\title{
INVENTARIO DE LAS PLANTAS VASCULARES Y TIPOS DE VEGETACIÓN DEL SANTUARIO EL Palmito, Sinaloa, MÉxico
}

\author{
Vascular flora and vegetation of Santuario El Palmito, Sinaloa, Mexico
}

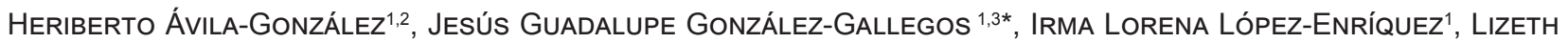
RuACHO-GonzÁleZ ${ }^{1}$, JACIEL RUBIO-CARDOZA ${ }^{1,2}$ y ARTURO CASTRO-CASTRO ${ }^{1,3}$

${ }^{1}$ Instituto Politécnico Nacional, Centro Interdisciplinario de Investigación para el Desarrollo Integral Regional, Unidad Durango, Durango, Durango, México.

${ }^{2}$ Maestría en Ciencias en Gestión Ambiental, CIIDIR-Durango, IPN, México.

${ }^{3}$ Cátedras CONACYT, México.

*Autor de correspondencia: xanergo@hotmail.com

\section{Resumen}

Antecedentes: El Santuario El Palmito es parte de una de las regiones terrestres prioritarias de México. Destaca por su diversidad de aves y alberga a uno de los bosques mesófilos más norteños en la vertiente del Pacífico. Sin embargo, carece de un inventario sistemático de su flora.

Preguntas: ¿Cuáles son los tipos de vegetación y sus elementos característicos en el Santuario? ¿Cuántas y cuáles familias, géneros y especies de plantas vasculares alberga? ¿Cuáles son las formas biológicas presentes en el área? ¿Existen especies bajo alguna categoría de protección?

Especies de estudio: Plantas vasculares.

Sitio de estudio y años de estudio: Santuario El Palmito, Sinaloa, México; octubre 2017 a octubre 2018.

Métodos: Se realizaron muestreos de la flora cada mes durante un año. Los ejemplares se identificaron con ayuda de literatura especializada, taxónomos especialistas y cotejo con ejemplares de herbario. El inventario se complementó con algunas bases de datos en línea y especímenes de herbario.

Resultados: Se registraron 492 especies, 285 géneros y 103 familias. Asteraceae, Fabaceae, Orchidaceae, Poaceae, Fagaceae y Lamiaceae fueron las familias con mayor riqueza. Se obtuvieron 53 registros nuevos de plantas vasculares para Sinaloa. Las formas biológicas predominantes fueron las hierbas (370 spp.), luego los arbustos (73) y los árboles (60). Por otra parte se registraron 14 especies (2.8\%) en alguna de las categorías de riesgo de la NOM-059-SEMARNAT-2010 y la UICN.

Conclusiones: El alto porcentaje de registros nuevos para la flora sinaloense pone de manifiesto la necesidad de continuar con las exploraciones en la entidad.

Palabras clave: Chara pinta, Concordia, flora vascular, Sierra Madre Occidental, Sinaloa.

\section{Abstract}

Background: El Palmito Sanctuary is part of one of the terrestrial priority regions from Mexico. It stands out for its diversity of birds, and hosts one of northernmost cloud mountain forests in the Pacific slope. However, it lacks a systematic inventory of its flora.

Questions: What are the types of vegetation and their characteristic elements in the Sanctuary? How many and which families, genera and species of vascular plants are host? What are the biological forms present in the area? Are there species under any protection category?

Species of study: Vascular plants.

Study site and years of study: El Palmito Sanctuary, Sinaloa, Mexico; October 2017 to October 2018.

Methods: Sampling of the flora was done every month for one year. The specimens were identified with the help of specialized literature, specialized taxonomists and comparison with herbarium specimens. The inventory was complemented with online databases and herbarium specimens.

Results: 492 species, 285 genera and 103 families were registered. Asteraceae, Fabaceae, Orchidaceae, Poaceae, Fagaceae and Lamiaceae were the richest families. 53 new records are reported for Sinaloa. The predominant live forms were herbs $(370 \mathrm{spp}$.), then shrubs $(73)$ and trees (60). Moreover, 14 species (2.8\%) were registered in one of the risk categories of NOM-059-SEMARNAT-2010 and IUCN.

Conclusions: The high percentage of new records for the Sinaloa's flora indicates the need to continue with the explorations in the entity. Key words: Chara pinta, Concordia, Sierra Madre Occidental, Sinaloa, vascular flora. 
El Santuario El Palmito (municipio de Concordia, Sinaloa), también conocido como Reserva La Chara Pinta, fue propuesto el 5 de junio de 2008 con carácter de Área Natural Protegida (ANP) estatal de tipo Santuario (SEMARNAT 2008). Las características más relevantes del sitio que determinan la categoría de Santuario son: 1) presencia de bosque mesófilo de montaña, 2) alberga la población más importante de la chara pinta (Cyanocorax dickeyi) y 3) es zona de distribución del jaguar (Panthera onca), ocelote (Leopardus pardalis), onza (Puma yagouaroundi) y puma (P. concolor), diversas especies de murciélagos, roedores y ungulados, como el venado cola blanca (Odocoileus virginianus), y el pecarí de collar (Pecari tajacu); así como reptiles y anfibios, algunos de ellos en alguna categoría de riesgo. Forma parte de las primeras iniciativas de protección que se desarrollan en el estado de Sinaloa y constituye un sitio importante para la conservación de la vida silvestre en la Sierra Madre Occidental (SMO), ya que se han registrado más de 120 especies de aves, 57 de mamíferos y 146 de flora (CONANP 2008, López-Segoviano et al. 2019). En el caso de las aves, destaca por ser un sitio de distribución, alimentación y reproducción, tanto para especies residentes como migratorias. Un ejemplo es la chara pinta ( $C$. dickeyi), especie endémica y amenazada cuyo rango de distribución no alcanza más de $100 \mathrm{~km}$ de ancho y $300 \mathrm{~km}$ de largo en los estados de Durango, Nayarit y Sinaloa (CONANP 2008, López-Segoviano et al. 2019). Debido a estas características y al estado de conservación de los bosques tropicales de la cuenca del río El Salto, ArriagaCabrera et al. (2000) consideraron a esta área como parte de la Región Terrestre Prioritaria Río Presidio (RTP55).

La vegetación dominante en el Santuario se conforma por bosque de pino-encino (BPE) y bosque mesófilo de montaña (BMM), los cuales en conjunto son los que poseen una mayor cantidad de especies y especies endémicas en México (Rzedowski 1996, Márquez-Linares \& González-Elizondo 1998, Alcántara-Ayala \& Luna-Vega 2001, Espejo-Serna 2014). El BPE es la vegetación dominante en la SMO, se presenta en ambientes muy variables y comprende diferentes asociaciones que abarcan todos los grados intermedios desde el pinar al encinar puro (González-Elizondo et al. 2007, 2012b), peculiaridad que hace muy difícil su determinación (Rzedowski 1978). En sitios con alta humedad ambiental se desarrollan parches de bosque de encino-pino intermedios con el BMM (González-Elizondo et al. 2007, 2012b). En estos bosques mixtos de la SMO destacan los madroños (Arbutus tessellata y A. xalapensis), pinos (Pinus devoniana, $P$. herrerae y $P$. maximinoi), capulines (Prunus serotina) y encinos (Quercus crassifolia, $Q$. scytophylla y $Q$. viminea), así como especies de los géneros Alnus, Garrya y Cornus, que son otros componentes de sitios con mayor humedad ambiental (MárquezLinares \& González-Elizondo 1998, Beltrán 2003b, González-Elizondo et al. 2007, 2012b).

El BMM posee una estructura, afinidad florística y composición de especies bastante diversas (Rzedowski 1978). Pese a ocupar una extensión reducida en México, el BMM es el ecosistema que alberga la mayor diversidad de especies de flora por unidad de superficie (Rzedowski 1996, AlcántaraAyala y Luna-Vega 2001, Villaseñor 2010). Una causa de su riqueza es la mezcla de elementos de las regiones Neártica y Neotropical. La composición, fisonomía y estructura del BMM varía de acuerdo a su ubicación geográfica y altitudinal y por sus características climáticas (Luna-Vega et al. 1994, Alcántara-Ayala \& Luna-Vega 2001, Ponce-Vargas et al. 2006, López-Pérez et al. 2011). Se señala que el BMM en Sinaloa es aún más restringido que en el contexto nacional y posee una riqueza florística baja en comparación con el resto (Rzedowski 1978). En ellos sobresale un dosel con árboles de afinidad neártica, compuesto por abetos (Abies), capulines (Prunus), carpes, moras de la sierra o pipinques (Carpinus y Ostrya), corpos (Magnolia), encinos (Quercus), fresnos (Fraxinus), pinos (Pinus) y tilas (Tilia), así como elementos neotropicales, como aguacates (Persea), arrayanas o guayabillos (Eugenia), cedros (Cedrela) y la mano de león (Oreopanax) (Beltrán 2003a, González-Elizondo et al. 2007).

Aunque existen varios estudios en los bosques de la SMO, aún quedan muchos vacíos de información (González-Elizondo et al. 2007, 2012b, 2017). Además, los inventarios florísticos en Durango y Sinaloa son relativamente escasos en comparación con otros estados. En un mapa en que Villaseñor (2016) representa la ubicación y extensión de los inventarios florísticos principales desarrollados en el país, se muestran siete para Durango y tres para Sinaloa. En contraste, Chiapas, Coahuila, Estado de México, Guerrero, Hidalgo, Jalisco, Michoacán, Oaxaca, Puebla y Veracruz al menos cuentan con 10. Este sesgo puede deberse en parte a una tradición botánica mucho más antigua y arraigada en el centro y sur del país, así como al área de influencia histórica y distribución de las instituciones que desarrollan el mayor porcentaje de investigación en biodiversidad. Por otra parte, se han descubierto 64 especies nuevas de plantas vasculares en la SMO de 2010 a la fecha (Henrickson \& Van Devender 2010, Henrickson et al. 2011, Mayfield \& Steinmann 2010, González-Tamayo \& Hernández-Hernández 2010, JimenoSevilla \& Carrillo-Reyes 2010, Nesom 2010, 2014, 2017, 2018, Saarela et al. 2010, Sánchez-Ken 2010, Estrada-C et al. 2011, 2016, Ramírez-Noya et al. 2011, Turner 2011, 2012, 2013a, 2013b, 2013c, 2015, González-Elizondo et al. 2012a, Reyes-Santiago et al. 2012a, 2012b, 2015, 2017, Van Devender \& Nesom 2012, Borhidi et al. 2013, Cruz-Durán \& Sousa 2013, 2017, González-Gallegos 2013, 2016, González-Gallegos \& López-Enríquez 2016, 2017, Rodríguez \& Ortiz-Catedral 2013, Anderson 2014, Costea et al. 2014, Folk \& Freudenstein 2014, Spellenberg 2014, Steinmann 2014, Castro-Castro et al. 2015, Wallnofer 2015, Mcdonnell \& Fishbein 2016, Pérez-Cálix 2016, Ruiz-Sánchez \& Castro-Castro 2016, Ruiz-Sánchez et al. 2017, Rzedowski 2016, García-Martínez et al. 2017, Semple 2017, Ahlquist \& Vincent 2018, Cohen 2018, González-Rocha et al. 2018, Gutiérrez-Ortega et al. 2018, Gutiérrez-Sánchez et al. 2018, Hernández-Barón et al. 2018, Lorence et al. 2018, Scheinvar et al. 2018, Zamudio et al. 2018), esto tomado con referencia al polígono de SMO propuesto por González-Elizondo et al. (2012b), y de éstas, seis se encontraron justo en el área del Santuario El Palmito (González-Elizondo et al. 2012a, Borhidi et al. 2013, Turner 2013a, Lorence et al. 2018, González-Rocha et al. 2018, Ávila-González et al. 2019). De 
igual manera, es probable que así como se han descubierto especies nuevas, conforme se incremente la exploración florística en la región se amplíe la distribución conocida de otras. Por tanto, lo anterior demuestra lo relevante que es priorizar esfuerzos para inventariar la biodiversidad de áreas estratégicas dentro de la SMO con la finalidad de reforzar el conocimiento de la biota del país. Esto es aún más relevante dado que la SMO es el macizo montañoso más extenso y el principal reservorio de bosques templados de México (González-Elizondo et al. 2012b).

El Santuario El Palmito constituye en particular un área de interés florístico debido a que se encuentra justo en la transición de los bosques templados y tropicales de la SMO, a su biodiversidad peculiar que se ve reflejada en la riqueza de su avifauna y en el descubrimiento reciente de especies nuevas de plantas vasculares y, por albergar parte de los BMM más norteños. Cabe destacar que existe una demanda local sobre la publicación del inventario de la flora del área por parte de los ejidatarios. Para ellos es importante conocer las plantas presentes a fin de proporcionar información a los visitantes y para complementar las observaciones y monitoreo de aves que realizan (Santos-Vázquez com. pers.). Por ejemplo, para señalar sobre que plantas perchan o se alimentan las aves. Debido a lo anterior, aquí se describen los tipos de vegetación del área y se presenta el inventario de la flora vascular del Santuario El Palmito; a través del mismo, se hace una contribución al conocimiento de la riqueza florística de Sinaloa y de la SMO, y se aporta información sobre su composición florística, las formas de crecimiento y especies que se encuentran bajo algún estatus de conservación.

\section{Materiales y métodos}

Área de estudio. El Santuario El Palmito tiene una superficie de 1,150 ha, y se localiza en el declive occidental de la SMO, en la comunidad El Palmito, Concordia, Sinaloa, justo en el límite con Durango, aunque una parte pequeña del polígono está en Durango (Figura 1). Presenta un intervalo altitudinal que va de los 1,440 hasta los 2,780 m snm. El área se caracteriza por tener un relieve con pendientes abruptas que dan paso a cañadas protegidas por paredones de roca volcánica. En el área se forman varios arroyos intermitentes durante la época de lluvias, uno de los más importantes es La Tolva que abastece de agua a la comunidad. El clima es templado

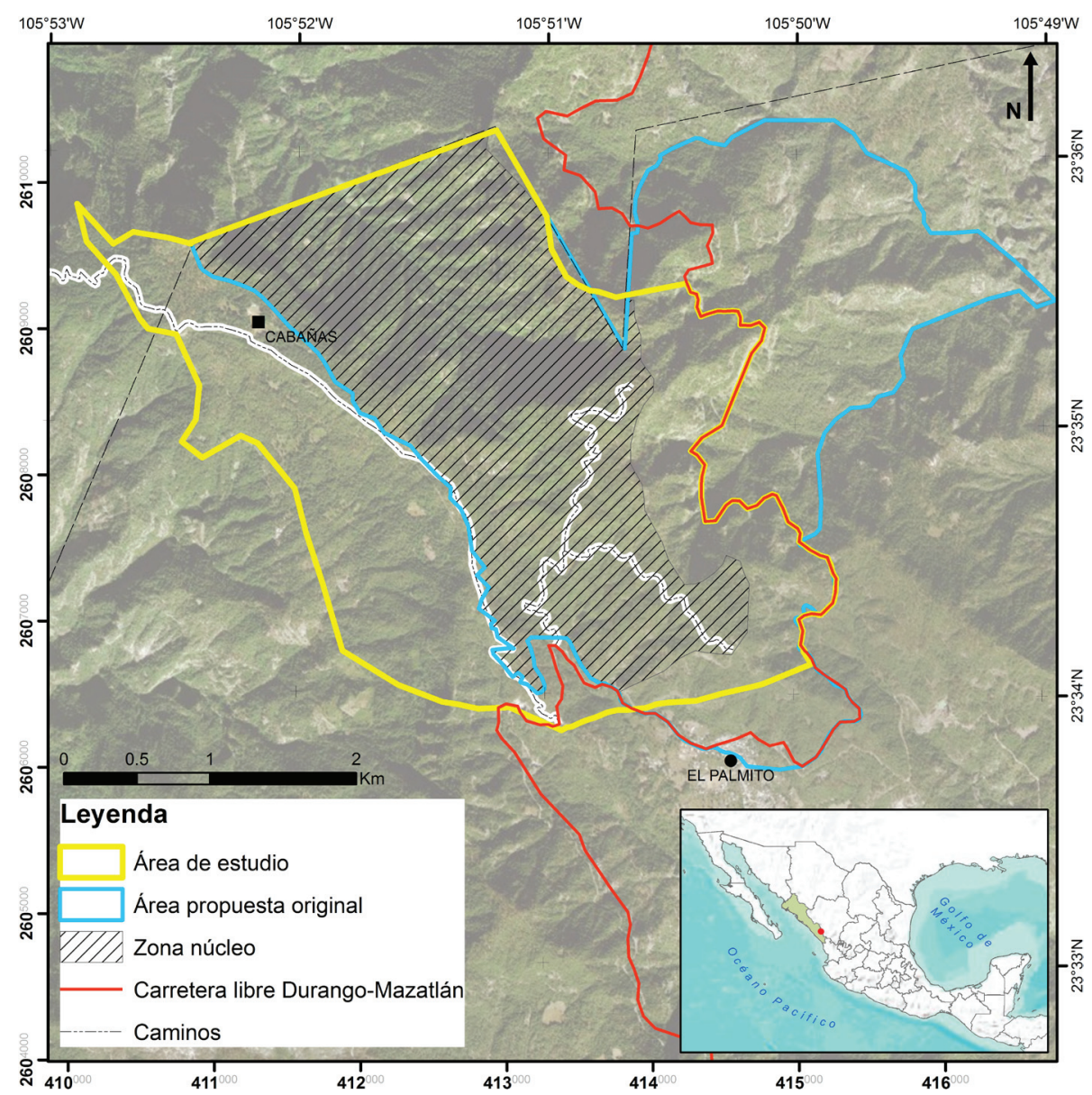

Figura 1. Localización del Santuario EL Palmito, su zona núcleo, y el área de estudio. 
subhúmedo con lluvias en verano e invierno [S (w1 )(w)], la temperatura media anual es de $16{ }^{\circ} \mathrm{C}$, y la precipitación total anual es de 1,246 mm, el suelo se compone por Regosol y Litosol principalmente (CONANP 2008, López-Segoviano et al. 2019).

El polígono que se estableció para realizar el inventario, no corresponde con el definido y propuesto por CONANP (2008). Por una parte, el área se amplió a fin de abarcar también la zona de cabañas y de senderos para los visitantes. Además, se excluyeron algunas porciones debido a su inaccesibilidad en función del relieve. El polígono final comprende una superficie de 1,150 ha, en la que se incluye la zona núcleo completa (808.44 ha; Figura 1). No obstante, al considerar que el área de estudio incluye todos los tipos de vegetación de la ANP y a su proporción de superficie, el inventario generado es un referente de la flora presente en la totalidad de la reserva.

Trabajo de campo. Se establecieron 10 puntos fijos al azar con distancias uniformes entre sí a manera de referencia para cubrir los diferentes tipos de vegetación del área de estudio, y así asegurar que durante las visitas se recorriera toda el área. Posterior a esto, se realizaron 13 visitas por un periodo de cinco a siete días, una vez por mes durante el lapso de octubre de 2017 a octubre de 2018. En cada exploración la colecta se realizó en cada uno de los puntos definidos y en los trayectos entre ellos. Se colectaron tres duplicados por cada especie cuando fue posible, un juego completo de colectas se depositó en el herbario CIIDIR, y los restantes serán enviados a los herbarios de la Universidad Autónoma de Sinaloa (UAS) y al de la Universidad Nacional Autónoma de México (MEXU). La recolección y herborización de los ejemplares se realizó de acuerdo a las especificaciones de Lot \& Chiang (1986).

Caracterización de la vegetación. Los tipos de vegetación se definieron y caracterizaron de acuerdo a la propuesta de González-Elizondo et al. (2007), una adaptación regional referida al trabajo Vegetación de México (Rzedowski 1978). Se combinaron criterios físonómicos, ecológicos y florísticos. El proceso se realizó mediante observaciones directas en campo y descripciones cualitativas. La composición florística de cada uno de los tipos de vegetación se obtuvo a partir de los ejemplares colectados e identificados.

Trabajo de escritorio. La identificación del material colectado se realizó con el apoyo de claves y trabajos taxonómicos (floras, monografías, revisiones taxonómicas), cotejo con ejemplares botánicos del herbario CIIDIR, herbarios virtuales y apoyo de taxónomos especialistas. Adicionalmente, se revisaron bases de datos en SEINet (2018), Naturalista (2018) y el herbario CIIDIR, para identificar colectas realizadas en el área. En el caso de estas fuentes adicionales, una vez extraídos los registros se consideraron algunos criterios para decidir su pertinencia en el listado: 1) que los ejemplares estuvieran determinados por un especialista del grupo (cuando fue posible se hizo la verificación con base en imágenes digitales o ejemplares de herbario), 2) que correspondieran sin duda al área de la ANP y 3) que fuera congruente respecto a la distribución de las especies. Toda la información de las colectas realizadas y consultadas se integró en una matriz de datos.

Para clasificar en formas de vida a las especies encontradas se siguió la propuesta de Frías-Castro et al. (2013), en ella se consideran los siguientes tres atributos: hábito (árbol, arbusto, bejuco o liana, hierba, rosetófila y globosa), hábitat (epífito, rupícola y terrestre) y tipo de nutrición (autótrofa, hemiparásita, holoparásita y saprófita). Además se consideró la duración del ciclo de vida (anual y perenne). En este aspecto, se siguieron las definiciones de Font-Quer (1953), Moreno (1984) y Harris \& Harris (1994). Para ayudar en la designación de los criterios de formas biológicas se cotejaron las descripciones originales de las plantas y la base de datos del herbario CIIDIR. Se identificaron las especies bajo alguna categoría de conservación listada por la Norma Oficial Mexicana (SEMARNAT 2010) y la Unión Internacional para la Conservación de la Naturaleza (IUCN 2019).

Listado florístico. Las plantas en el listado se ordenaron por grandes grupos: helechos y licofitas (Mickel \& Smith 2004), gimnospermas (Christenhusz et al. 2011) y angiospermas (magnolídeas, monocotiledóneas y eudicotiledóneas; APG 2016); y las autoridades nomenclaturales fueron designadas de acuerdo a lo establecido en las bases de datos Tropicos (2018-2019) e IPNI (2019). Dentro de cada uno de los grupos anteriores se siguió una secuencia alfabética para familias, géneros, especies y taxones infraespecíficos. Para cada taxon se proporciona la forma biológica, (hábito, hábitat, tipo de nutrición y duración del ciclo de vida), siglas de los colectores y sus números de colecta. También se señalan las especies nuevas, endémicas al área y los registros nuevos para Sinaloa.

\section{Resultados}

Vegetación. En el área predomina el BPE (Figura 2A), aunque también existen algunos relictos de BMM (Figura 2B), en cañadas y laderas protegidas. No obstante, en ocasiones los dos tipos de vegetación se mezclan, lo que dificulta su delimitación, a la vez que incrementan la diversidad local debido al traslape de las especies propias a cada uno de esos ecosistemas.

En las áreas donde domina el BPE el estrato arbóreo alcanza 10 a 15 metros de altura y se registran a Alnus jorullensis subsp. jorullensis, Arbutus xalapensis, Pinus devoniana, P. douglasiana, P. herrerae (Figura 3E), Prunus serotina, Quercus calophylla, Q. convallata, Q. fulva (Figura 3B), $Q$. laeta, $Q$. reticulata y $Q$. viminea. La presencia de plantas hemiparásitas en este estrato es recurrente, representadas por Cladocolea cupulata, Phoradendron longifolium y Psittacanthus calyculatus. En las áreas de mayor elevación se observan a Arbutus bicolor, Pinus durangensis, $P$. strobiformis, Quercus brachystachys y Q. rugosa. En algunas áreas el estrato arbustivo y herbáceo son dominadas por Cestrum thyrsoideum y Pteridium aquilinum, además es común encontrar especies de Roldana ( $R$. barba-johan- 

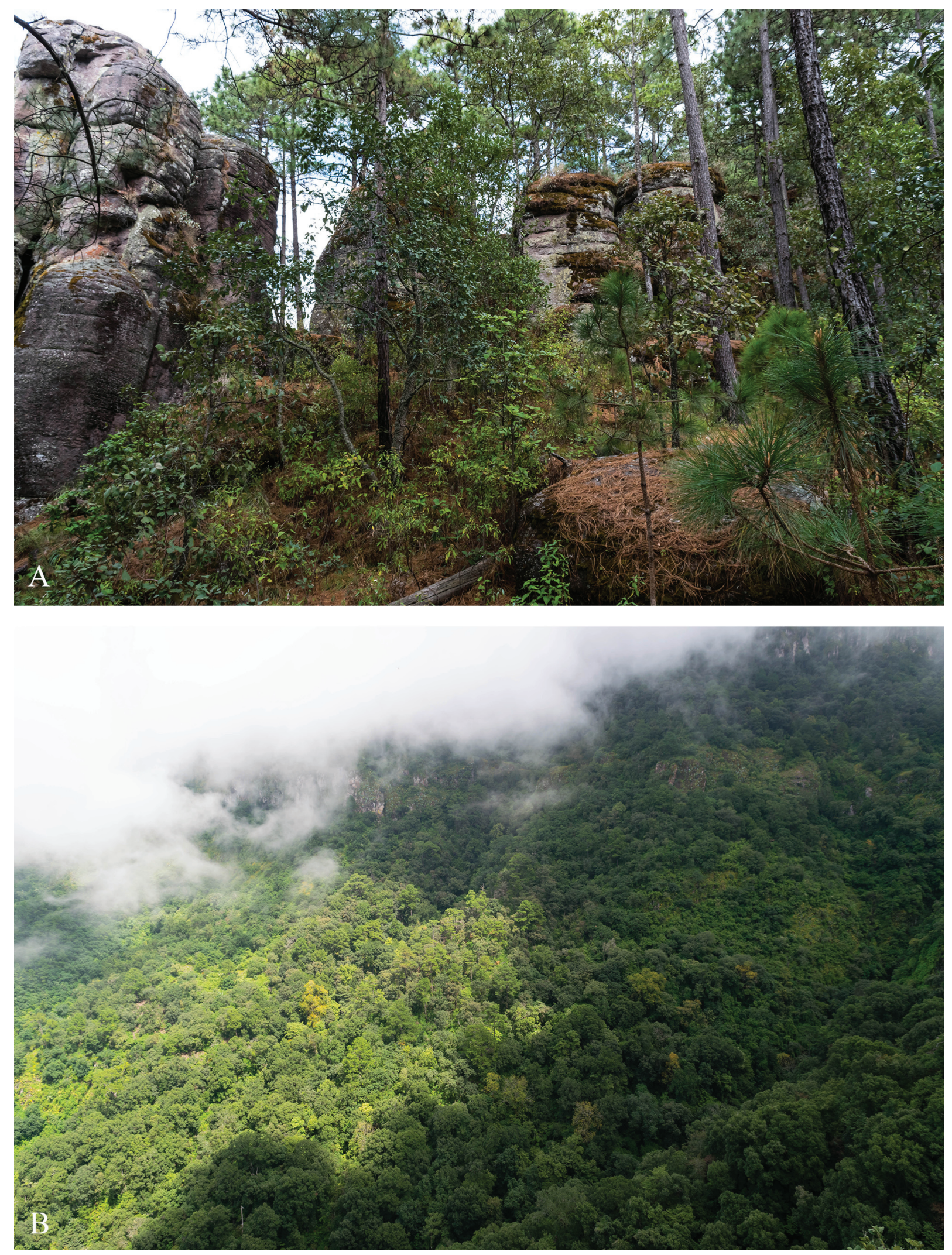

Figura 2. Vegetación del Santuario El Palmito. A) Bosque de pino-encino y B) Bosque mesófilo de montaña. 

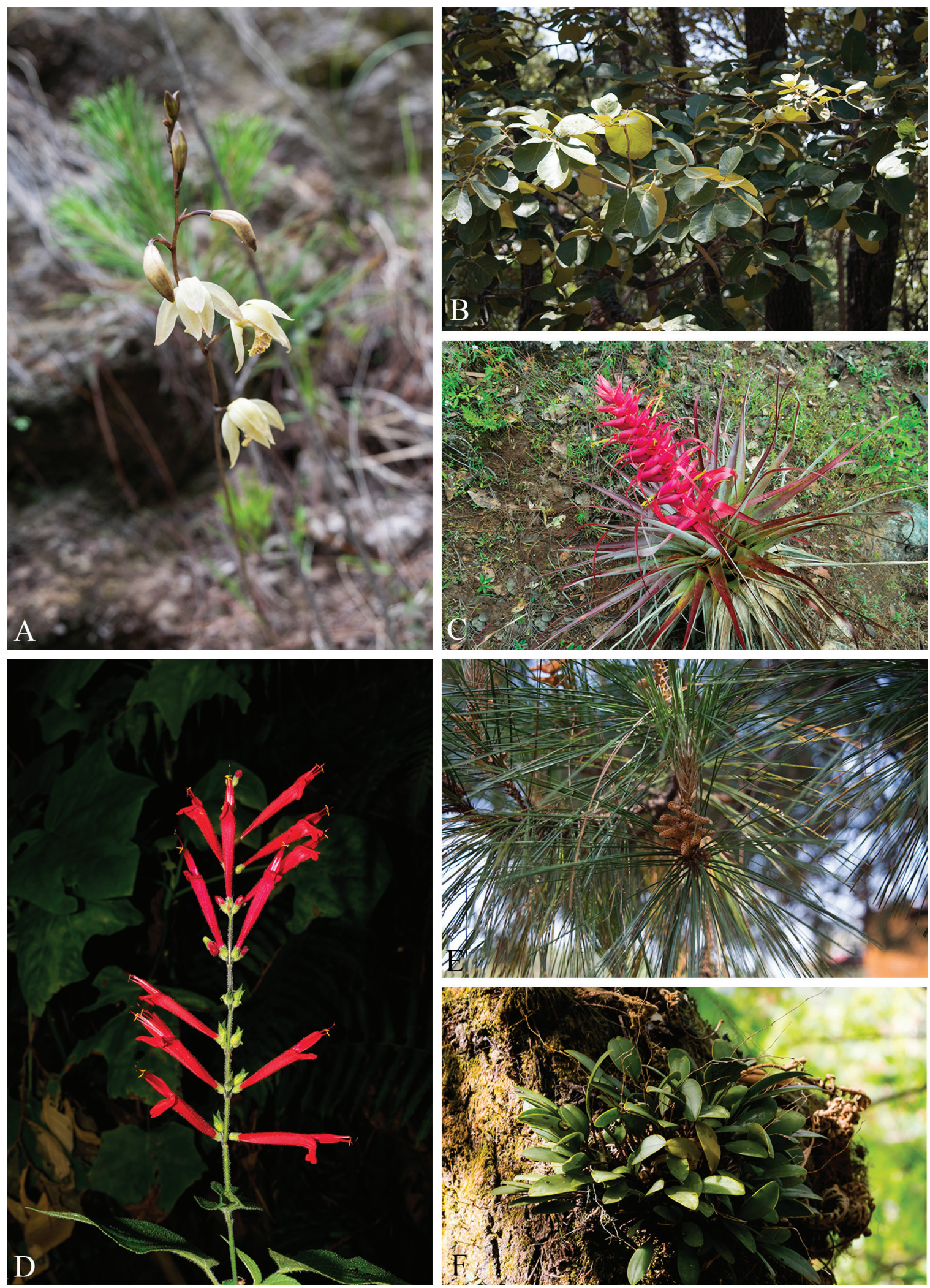

Figura 3. Algunas especies representativas del bosque de pino-encino. A) Bletia villae, B) Quercus fulva, C) Tillandsia borealis, D) Salvia elegans, E) Pinus herrerae y F) Stelis xerophila. 
Flora vascular de El Palmito, Sinaloa, México

Tabla 1. Diversidad de la flora vascular del Santuario El Palmito, municipio de Concordia, Sinaloa, México.

\begin{tabular}{lccccc}
\hline Grupo & Familias & Géneros & Especies & Subespecies & Variedades \\
\hline Licofitas & 2 & 2 & 2 & 0 & 0 \\
Helechos & 9 & 21 & 35 & 0 & 1 \\
Gimnospermas & 2 & 3 & 9 & & \\
Angiospermas & & & 4 & 2 & 0 \\
Magnolídeas & 3 & 3 & 93 & 13 & 1 \\
Monocotiledóneas & 16 & 201 & 349 & 15 & 14 \\
Eudicotiledóneas & 71 & 285 & 492 & & 19 \\
Total & 103 & & & & \\
\hline
\end{tabular}

nis, R. hartwegii y R. jurgensenii). Los géneros Castilleja, Desmodium, Ipomoea, Muhlenbergia, Salvia y Solanum también están bien representados.

En los manchones de BMM domina un estrato arbóreo de 10 a 20 metros representado por Abies neodurangensis, Cedrela odorata, Meliosma dentata, Pinus herrerae, $P$. maximinoi, Quercus calophylla, Q. grahamii (Figura 4F), $Q$. scytophylla, $Q$. splendens, Styrax ramirezii y Tilia americana; otras especies comunes son Arbutus xalapensis, Dendropanax arboreus, Magnolia tarahumara (Figura 4E) y Prunus serotina subsp. serotina (Figura 4A). En un estrato más bajo (7 a 10 metros) dominan Cercocarpus macrophyllus, Clethra hartwegii, Cornus disciflora, Eugenia crenularis (Figura 4B), Fuchsia arborescens, Oreopanax echinops, O. peltatus, Ternstroemia lineata y Urera corallina. Algunas especies como Arisaema macrospathum (Figura 4G), Begonia biserrata, Botrychium virginianum, Chamaedorea pochutlensis, Deppea guerrerensis, Goodyera striata, Greenwoodiella micrantha var. garayana, Guardiola mexicana var. mexicana, Hoffmannia cuneatissima y Peltostigma eximium (Figura 4C), se restringen a este tipo de vegetación. Las epífitas están representadas por varias especies de helechos (Asplenium cuspidatum, Campyloneurum angustifolium, Elaphoglossum erinaceum var. occidentale, E. mulleri, E. petiolatum y Pleopeltis angusta), bromelias (Tillandsia borealis, T. bourgaei, T. macdougallii) y orquídeas (Corallorhiza maculata, Goodyera striata, Greenwoodiella micrantha var. garayana, Habenaria macvaughiana, Isochilus bracteatus, Malaxis brachyrrhynchos, M. maianthemifolia, Prosthechea squalida y Rhynchostele cervantesii).

Diversidad florística. Se obtuvieron 687 números de colecta (en la figura 5 se muestra la distribución de los puntos de colecta), y se conformó una matriz de datos con 870 registros derivados de las colectas y de la consulta de literatura especializada y de bases de datos (CIIDIR, SEINet y Naturalista). Se registraron 103 familias, 285 géneros y 492 especies (Apéndice 1). El grupo mejor representado fue el de las angiospermas con 446 especies $(90.65 \%)$, seguido de los helechos con $35(7.11 \%)$, gimnospermas con $9(1.82 \%)$ y licofitas con $2(0.40 \%)$ (Tabla 1$)$. Las familias más ricas fueron Asteraceae (75 spp.; $15.24 \%$ ), Fabaceae (36 spp.; $7.31 \%$ ), Orchidaceae (30 spp.; $6.09 \%$ ), Poaceae (19 spp.;
$3.86 \%$ ), Fagaceae (15 spp.; $3.04 \%$ ), Lamiaceae (16 spp.; $3.25 \%$ ), Orobanchaceae (14 spp.; $2.84 \%$ ), Rubiaceae (12 spp.; $2.43 \%$ ), Cyperaceae y Polypodiaceae (10 spp.; $2.03 \%$ ). El resto de las familias agrupan 255 especies, lo que representa el $51.82 \%$ (Tabla 2). Las familias más ricas en géneros fueron Asteraceae con 45 (15.78\%), Fabaceae y Orchidaceae con 17 (5.96\%), Poaceae con 10 (3.50\%), Rubiaceae con 8 (2.86\%), Asparagaceae con 7 (2.45\%), Orobanchaceae, Lamiaceae y Pteridaceae con 6 (2.10\%), Rosaceae y Plantaginaceae con 5 (1.75\%), cada una. Las 92 familias restantes contienen 154 géneros, que representan el 54.03\% (Tabla 2). Los géneros más diversos fueron Quercus (15 spp.), Salvia (11), Muhlenbergia (9) y Castilleja (7).

Forma biológica. El hábito dominante es el de las hierbas (370 especies, $75.20 \%$ ), seguido por arbustos (73; $14.83 \%$ )

Tabla 2. Familias con más riqueza de géneros y especies (columnas de la izquierda) y géneros con más especies (derecha) en El Santuario El Palmito

\begin{tabular}{lcclc}
\hline Familias & Géneros & Especies & Género & Especies \\
\hline Asteraceae & 45 & 75 & Quercus & 15 \\
Fabaceae & 17 & 36 & Salvia & 11 \\
Orchidaceae & 17 & 30 & Muhlenbergia & 9 \\
Poaceae & 10 & 19 & Castilleja & 7 \\
Fagaceae & 7 & 15 & Pinus & 6 \\
Asparagaceae & 7 & 9 & Cyperus & 6 \\
Lamiaceae & 6 & 16 & Stevia & 6 \\
Rubiaceae & 8 & 12 & Ipomoea & 6 \\
Orobanchaceae & 5 & 13 & Desmodium & 6 \\
Cyperaceae & 5 & 10 & Solanum & 6 \\
Polypodiaceae & 5 & 10 & Malaxis & 5 \\
Pteridaceae & 4 & 7 & Aldama & 5 \\
Solanaceae & 4 & 10 & Bidens & 5 \\
Dryopteridaceae & 3 & 7 & Cosmos & 5 \\
Onagraciae & 3 & 7 & Roldana & 5 \\
Pinaceae & 3 & 7 & & \\
\hline
\end{tabular}



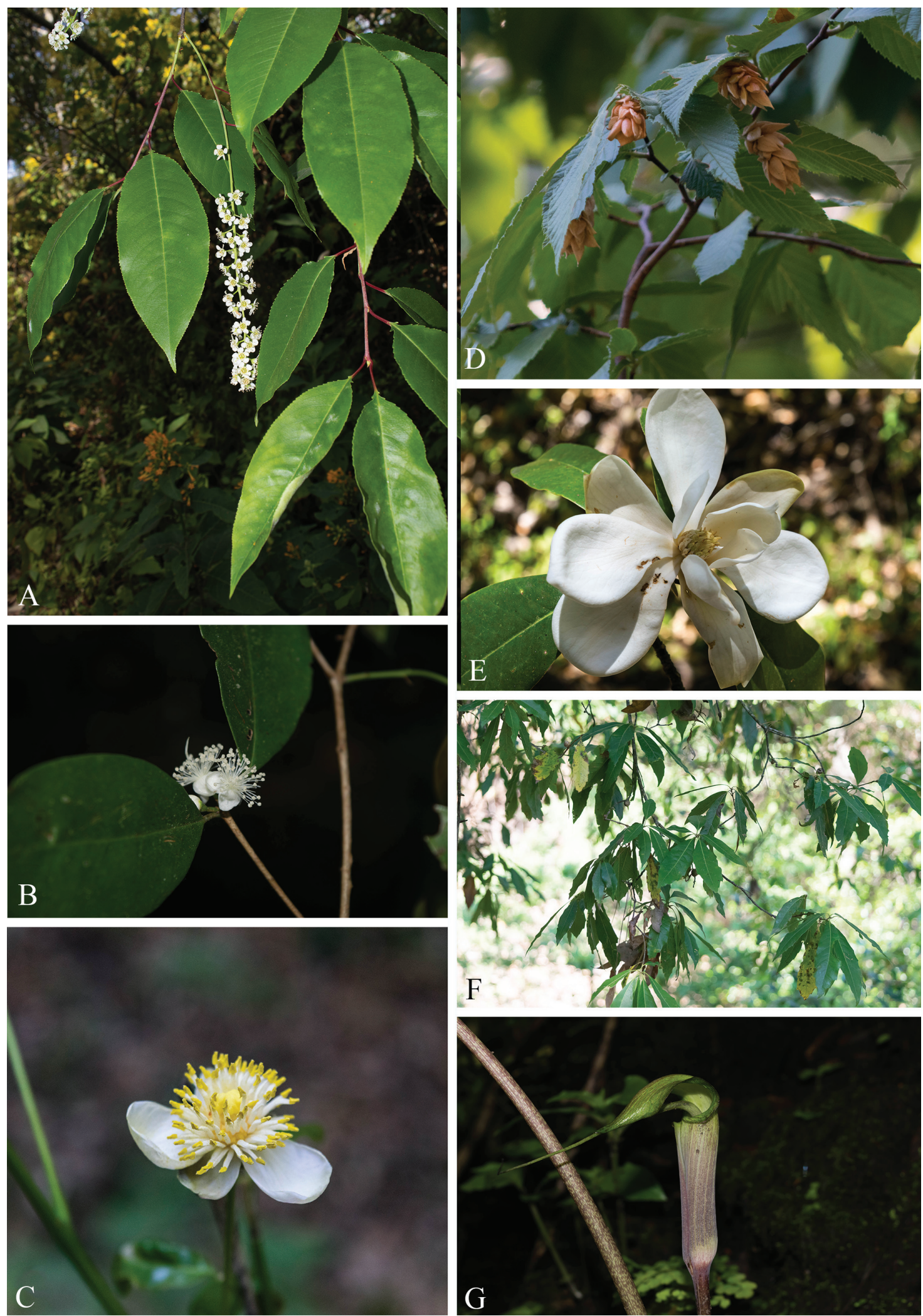

Figura 4. Algunas especies representativas del bosque mesófilo de montaña. A) Prunus serotina, B) Eugenia crenularis, C) Peltostigma eximium, D) Ostrya virginiana, E) Magnolia tarahumara, F) Quercus grahamii y G) Arisaema macrospathum. 


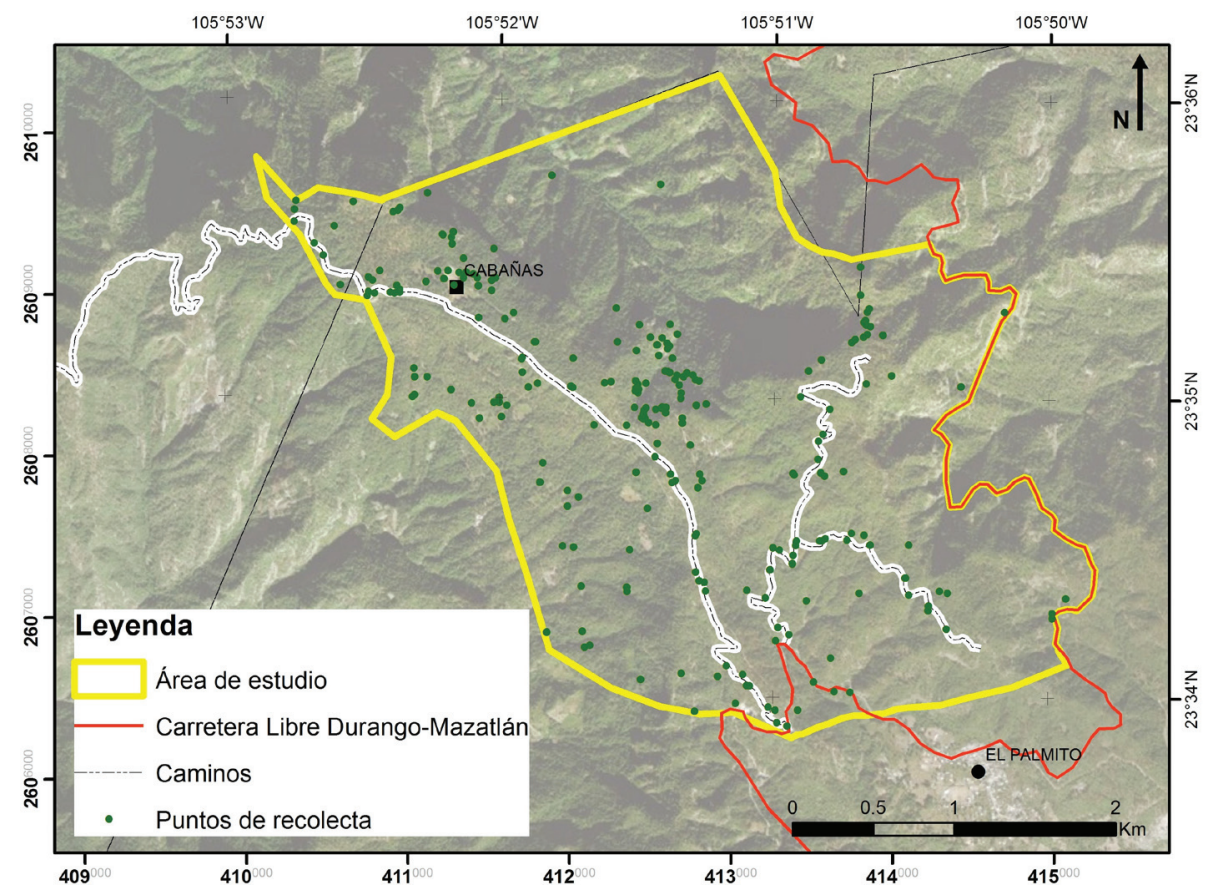

Figura 5. Distribución de los puntos de colecta dentro del área de estudio.

y árboles $(60 ; 12.52 \%)$. En contraste, los menos representados son las rosetófilas, globosas y bejucos $(5,2$ y 3 , respectivamente, $2.03 \%$ en conjunto). Las plantas dominantes, según el tipo de hábitat que ocupan, son las terrestres (449; $91.26 \%)$, después las rupícolas $(59 ; 11.99 \%)$ y las menos representadas son las epífitas $(31 ; 6.30 \%)$. De acuerdo al tipo de nutrición las plantas autótrofas son las más abundantes en el área $(472 ; 95.93 \%)$, luego las hemiparásitas $(18 ; 3.65 \%)$ y holoparásitas $(2 ; 0.40 \%)$. El ciclo de vida dominante es el perenne (467; $94.91 \%$ ) y después el anual (57; $11.58 \%$; Apéndice 1; Tabla 3). Algunas especies califican dentro de las dos categorías anteriores, por ello la suma de perennes y anuales no coincide con la riqueza total registrada.

Especies bajo categoría de riesgo. De las 492 especies registradas para el ANP, 14 de ellas (2.84\%) se incluyen en algunas categorías de riesgo de la NOM-059-SEMARNAT2010 (SEMARNAT 2010) o la Unión Internacional para la Conservación de la Naturaleza (IUCN 2019; Tabla 4). Solo Cedrela odorata se comparte en ambas listas (Tabla 4). Nueve especies están incluidas en algunas de las categorías de riesgo propuestas en la SEMARNAT (2010); una en peligro de extinción (P), tres amenazadas (A) y cinco sujetas a protección especial (Pr; Tabla 3). De las seis especies encontradas en las categorías de la IUCN (2019), cuatro están la categoría de vulnerable (VU) y dos en casi amenazadas (NT; Tabla 4).

En el área de estudio se desarrolla un conjunto de especies que no son consideradas en las listas de protección señaladas, pero que representan casos interesantes si se toma en cuenta su rareza o grado de endemismo. En estos casos se puede considerar a Lobelia macrocentron (Campanulaceae), la cual fue redescubierta en el área de estudio después de 169 años de su primer encuentro (Ávila-González et al. 2018) y una especie nueva del género Bletia (Orchidaceae) (Ávila-González et al. 2019). Hasta ahora, estos dos taxa sólo se conocen del Santuario El Palmito.

Tabla 3. Formas biológicas de la flora del Santuario El Palmito por hábito, hábitat, tipo de nutrición y duración del ciclo de vida. H (hierba), Ar (arbusto), A (árbol), R (rosetófila), B (bejuco), G (globosa), $\mathrm{T}$ (terrestre), Ru (rupícola), E (epífita), Au (autótrofa), He (hemiparásita), H (holoparásita), P (perenne), An (anual). Cabe señalar que los totales no coinciden con la cifra de especies registradas en el área de estudio debido a que algunas especies califican en más de uno de los atributos de formas biológicas aquí considerados.

\begin{tabular}{lcccccccc}
\hline $\begin{array}{l}\text { Hábito/ } \\
\text { Número } \\
\text { de especies }\end{array}$ & \multicolumn{2}{c}{ Hábitat } & \multicolumn{3}{c}{$\begin{array}{c}\text { Forma de } \\
\text { nutrición }\end{array}$} & \multicolumn{2}{c}{$\begin{array}{c}\text { Ciclo de } \\
\text { vida }\end{array}$} \\
\hline H (370) & T & Ru & $\mathbf{E}$ & Au & He & H & P & An \\
Ar (73) & 711 & 51 & 25 & 337 & 13 & 2 & 319 & 57 \\
Á (60) & 60 & 0 & 0 & 60 & 0 & 0 & 60 & 0 \\
R (5) & 3 & 3 & 1 & 5 & 0 & 0 & 5 & 0 \\
B (3) & 3 & 0 & 0 & 3 & 0 & 0 & 3 & 0 \\
G (2) & 0 & 2 & 0 & 2 & 0 & 0 & 2 & 0 \\
\hline Total & 449 & 60 & 31 & 474 & 18 & 2 & 470 & 57 \\
\hline
\end{tabular}


Tabla 4. Especies en alguna categoría de protección por la Unión Internacional para la Conservación de la Naturaleza (IUCN) y la Norma Oficial Mexicana NOM-059-SEMARNAT-2010. Pr (sujeta a protección especial), A (amenazada), P (en peligro de extinción), LC (preocupación menor), VU (vulnerable), NT (casi amenazada). No se consideró la categoría LC de la IUCN dado que aplica a especies que no son de interes o prioritarias para la conservación.

\begin{tabular}{lcc}
\hline Especies & IUCN & NOM-059-SEMARNAT-2010 \\
\hline Cedrela odorata & $\mathrm{VU}$ & $\mathrm{Pr}$ \\
Chamaedorea pochutlensis & & $\mathrm{A}$ \\
Cornus disciflora & $\mathrm{VU}$ & $\mathrm{P}$ \\
Litsea glaucescens & & $\mathrm{A}$ \\
Mammillaria senilis & & \\
Oreopanax echinops & $\mathrm{VU}$ & \\
Oreopanax peltatus & $\mathrm{VU}$ & $\mathrm{Pr}$ \\
$\begin{array}{l}\text { Ostrya virginiana } \\
\text { Pedicularis glabra }\end{array}$ & & $\mathrm{Pr}$ \\
Pinus durangensis & $\mathrm{NT}$ & \\
Pinus lumholtzii & $\mathrm{NT}$ & \\
Pinus strobiformis & & $\mathrm{Pr}$ \\
Rhynchostele cervantesii & & $\mathrm{A}$ \\
Tripsacum zopilotense & & $\mathrm{Pr}$ \\
\hline
\end{tabular}

Nuevos registros. Se encontraron 53 especies que no habían sido registradas para Sinaloa, de éstas, 16 no están reportadas en estados vecinos (Tabla 5; Apéndice 1). Por ejemplo, Roldana jurgensenii se conocía sólo de Chiapas y Oaxaca, y Gentiana mirandae de Hidalgo, Veracruz y Guerrero (Villarreal-Quintanilla et al. 2009, Villaseñor 2016, Tropicos 2018-2019).

\section{Discusión}

Vegetación. Los elementos arbóreos encontrados en el BPE son similares a los reportados por González-Elizondo et al. $(2007,2012 b)$ en los bosques mixtos con alta humedad ambiental en el declive occidental de la SMO. En estos sitios la mezcla de elementos del BPE y BMM es común. La combinación de las especies, tanto de pino como de encino, varía de acuerdo a las características del suelo y al gradiente altitudinal. Las combinaciones de Pinus durangensis, $P$. strobiformis, Quercus brachystachys y $Q$. rugosa entre los 2,450 y $2,700 \mathrm{msnm}$, Pinus douglasiana, $P$. devoniana, $P$. herrerae, P. maximinoi, P. lumholtzii, Quercus crassifolia, $Q$. fulva y $Q$. splendens entre otras especies de Quercus así como también Arbutus tesellata y A. xalapensis entre los 1,400 y 2,200 m snm, son muy similares a las reportadas para otros bosques de pino-encino de Sinaloa (Beltrán 2003b) y la SMO (González-Elizondo et al. 2007, 2012b). Además de que estas especies son señaladas como las más importantes de este tipo de vegetación (Beltrán 2003b).

El BMM de Sinaloa es menos diverso y complejo estructuralmente que los BMM del resto de México (Rzedows- ki 1978, Villaseñor 2010, González-Elizondo et al. 2012b, Gual-Díaz \& Rendón-Correa 2014). En el área de estudio se confirma esta tendencia ya que la riqueza encontrada en las porciones de BMM es menor a la registrada en estudios florísticos de bosques del sur y la vertiente oriental de México (Alcántara-Ayala \& Luna-Vega 2001, Espinosa-Jiménez et al. 2011, 2014, Gual-Díaz \& Rendón-Correa 2014), además de que no todos los géneros característicos del BMM están aquí representados (Rzedowski 1996). Estos bosques tanto en Durango como Sinaloa son de carácter relictual y distribución fragmentada (Rzedowski 1996, González-Elizondo et al. 2007). Algunos géneros de árboles cuantitativamente importantes en los BMM y presentes en El Santuario son: Alnus, Clethra, Cleyera, Cornus, Dendropanax, Fraxinus, Meliosma, Oreopanax, Prunus, Quercus, Styrax y Ternstroemia (Rzedowski 1996). Estos géneros, además de Ilex, Ostrya y Tilia, se consideran diagnósticos para delimitar y diferenciar esta comunidad (Rzedowski 1978, Villaseñor \& Gual-Díaz 2014), a su vez permiten diferenciarlo de los BPE, que se encuentran en el mismo piso altitudinal (López-Pérez et al. 2011). Otros elementos importantes del BMM son: Carpinus, Juglans, Platanus y Persea; sin embargo, estos no fueron encontrados en el área a pesar de que han sido reportados para el estado y que por su distribución natural pudieran estar allí.

Los resultados obtenidos ayudarán a tener un mejor entendimiento de los BMM de la porción norte de la vertiente del Pacífico, la información generada permitirá que sean considerados en análisis regionales o nacionales de este tipo de vegetación. Esto es relevante ya que los BMM de esta porción del país suelen ser ignorados o relegados a un segundo plano, situación evidente en los trabajos de CONABIO (2010) y Gual-Díaz \& Rendón-Correa (2014), en los cuales, aunque se mencionan éstos, no se cartografían de manera correcta ni se proporciona información detallada sobre los mismos.

Diversidad florística. Este trabajo representa el segundo esfuerzo para obtener un listado florístico del ANP, el primero fue el estudio justificativo para el establecimiento del Área Natural Protegida, el cual reportó 81 familias, 127 géneros y 173 especies (CONANP 2008), cifras menores a las reportadas en este trabajo, lo cual podría deberse a que el inventario provisto por CONANP derivó de la identificación de las especies mediante observaciones directas en campo, análisis de fotografías, algunos especímenes colectados y comparación de estos últimos con los materiales existentes en la colección de plantas del herbario de la Escuela de Biología de la Universidad Autónoma de Sinaloa.

En el Santuario El Palmito están presentes $2.11 \%$ de las especies, $9.98 \%$ de los géneros y $34.68 \%$ de las familias de plantas vasculares que Villaseñor (2016) enlista para México. En solo $11.5 \mathrm{~km}^{2}$ del territorio de Sinaloa $\left(58,200 \mathrm{~km}^{2}\right)$ está representado $51.5 \%$ de las 200 familias, $24.15 \%$ de los 1,180 géneros y $13.16 \%$ de las 3,736 especies registradas por Villaseñor (2016) para el estado. Al hacer una comparación de la riqueza global, por familias, géneros y especies respecto a inventarios florísticos con tipos de vegetación, área y rango altitudinal similar, destaca nuestra área de es- 
Tabla 5. Registros nuevos para la flora vascular del estado de Sinaloa, México. (**, Especies registradas en estados circunvecinos de Sinaloa; *, especies no registradas en estados circunvecinos de Sinaloa). Las abreviaturas de los estados se escriben conforme al ISO $3166-2$ de tres dígitos. ${ }^{a}$ La especie de Yucca aquí listada no ha podido ser identificada, lo que en consecuencia impide precisar su distribución; sin embargo, dado que el género se encuentra en estados circunvencinos a Sinaloa, fue catalogada como presente en estados vecinos.

\begin{tabular}{|c|c|c|}
\hline Especies & Distribución previa conocida & $\begin{array}{l}\text { Registro previo } \\
\text { en otros estados }\end{array}$ \\
\hline Asplenium castaneum & $\begin{array}{l}\text { CHP, CMX, DUR, GUA, GRO, JAL, MEX, MIC, MOR, NLE, OAX, PUE, QUE, TLA, } \\
\text { VER }\end{array}$ & $* *$ \\
\hline Blechnum occidentale & $\begin{array}{l}\text { CHP, COL, GRO, HID, JAL, MEX, MIC, MOR, NAY, OAX, PUE, QUE, SLP, TAB, } \\
\text { TAM, VER }\end{array}$ & $* *$ \\
\hline Bletia ensifolia & DUR, JAL, NAY, ZAC & $* *$ \\
\hline Bletia santosii & SIN & ** \\
\hline Bletia villae & DUR & $* *$ \\
\hline Corallorhiza maculata & $\begin{array}{l}\text { CHP, COA, COL, CMX, DUR, GUA, GRO, HID, JAL, MEX, MIC, MOR, NAY, NLE, } \\
\text { OAX, PUE, QUE, SON, TLA, VER, ZAC }\end{array}$ & $* *$ \\
\hline Cuphea tolucana & $\begin{array}{l}\text { CHP, COL, CMX, DUR, GRO, HID, } \\
\text { JAL, MEX, MIC, MOR, OAX, QUE, VER, ZAC }\end{array}$ & ** \\
\hline Deiregyne eriophora & $\begin{array}{l}\text { CHP, CHH, CMX, DUR, GRO, HID, JAL, MEX, MIH, MOR, NLE, OAX, PUE, SON, } \\
\text { TAM, TLA, VER }\end{array}$ & $* *$ \\
\hline Dichromanthus michuacanus & $\begin{array}{l}\text { AGU, CHP, CHH, COA, COL, CMX, DUR, GUA, GRO, HID, JAL, MEX, MIC, MOR, } \\
\text { NAY, NLE, OAX, PUE, QRO, SON, TLA, VER, ZAC }\end{array}$ & ** \\
\hline Digitaria filiformis & $\begin{array}{l}\text { AGU, CHP, CHH, COA, COL, CMX, DUR, GUA, GRO, JAL, MEX, MIC, MOR, NAY, } \\
\text { NLE, OAX, SON, TAM, VER, YUC, ZAC }\end{array}$ & $* *$ \\
\hline Donnellsmithia ternata & CHP, COA, DGO, JAL, NLE, OAX, SON, ZAC & $* *$ \\
\hline Drymaria leptophylla & $\begin{array}{l}\text { AGU, BCS, CHH, COL, CMX, DUR, GUA, HID, JAL, MEX, MIC, PUE, SLP, SON, TLA, } \\
\text { VER, ZAC }\end{array}$ & ** \\
\hline Gentiana mirandae & HID, VER, GRO & * \\
\hline Geranium lilacinum & COL, CMX, GRO, HID, JAL, MEX, MIC, MOR, OAX, QUE, SLP, TLA & * \\
\hline Greenwoodiella micrantha & GRO, JAL, MEX, MIC, MOR & * \\
\hline Habenaria macvaughiana & MEX, MIC & * \\
\hline Ipomoea decemcornuta & COL, JAL, OAX & * \\
\hline Isochilus bracteatus & COL, JAL, MEX, MIC, MOR, OAX & * \\
\hline Liparis madrensis & AGU, CHH, DUR, ZAC & $* *$ \\
\hline Lobelia macrocentron & DUR, NAY & ** \\
\hline Lupinus chihuahuensis & CHH, DUR & ** \\
\hline Lupinus exaltatus & COL, CMX, GRO, HID, JAL, MEX, MIC, MOR, OAX, PUE, TLA, VER & * \\
\hline Malaxis brachyrrhynchos & CHP, CMX, JAL, MEX, MIC, NAY y OAX & $* *$ \\
\hline Malaxis maianthemifolia & CHP, GRO, HID, MEX, MIC, MOR, OAX, PUE, QUE, SLP, TAM, VER & $*$ \\
\hline Malaxis pringlei & CHH, COL, DUR, JAL, NAY, ZAC & $* *$ \\
\hline Malaxis tepicana & JAL, NAY, ZAC & $* *$ \\
\hline Moussonia jaliscana & COL, DUR, JAL & $* *$ \\
\hline Muhlenbergia cenchroides & $\begin{array}{l}\text { BCS, CHP, CHH, COA, COL, CMX, GUA, GRO, HID, JAL, MEX, MIC, MOR, NAY, } \\
\text { NLE, OAX, PUE, QUE, SLP, TAM, TLA, VER }\end{array}$ & $* *$ \\
\hline Muhlenbergia michisensis & DUR, GRO & $* *$ \\
\hline Muhlenbergia quadridentata & $\begin{array}{l}\text { AGU, CHP, CHH, COA, COL, CMX, DUR, GUA, GRO, HID, JAL, MEX, MIC, MOR, } \\
\text { NLE, OAX, PUE, QUE, SLP, SON, TLA, VER, ZAC }\end{array}$ & $* *$ \\
\hline Muhlenbergia tenuifolia & $\begin{array}{l}\text { AGU, CHP, CHH, COA, COL, CMX, DUR, GUA, GRO, HID, JAL, MEX, MIC, MOR, } \\
\text { NAY, NLE, OAX, PUE, QUE, SLP, SON, TAB, TAM, TLA, VER, ZAC }\end{array}$ & $* *$ \\
\hline Oxalis divergens & $\begin{array}{l}\text { CHP, CHH, CMX, DUR, GUA, GRO, HID, JAL, MEX, MIC, OAX, PUE, QUE, SLP, } \\
\text { SON, TLA, VER }\end{array}$ & ** \\
\hline
\end{tabular}


Tabla 5. Registros

\begin{tabular}{|c|c|c|}
\hline Especies & Distribución previa conocida & $\begin{array}{l}\text { Registro previo } \\
\text { en otros estados }\end{array}$ \\
\hline Passiflora jorullensis & CHP, COL, GRO, JAL, MEX, MIC, NAY, OAX & ** \\
\hline Pedicularis glabra & COL, DUR, JAL, MIC & ** \\
\hline Peyritschia deyeuxioides & $\begin{array}{l}\text { AGU, CHP, CHH, COA, COL, CMX, DUR, GUA, GRO, HID, JAL, MEX, MIC, MOR, } \\
\text { NAY, NLE, OAX, PUE, QUE, SLP, SON, TAM, TLA, VER, ZAC }\end{array}$ & $* *$ \\
\hline Pinguicula parvifolia & COL, DUR, GRO, JAL, MEX, MIC, NAY, QUE, SLP, ZAC & ** \\
\hline Pitcairnia robert-downsii & DUR & ** \\
\hline Pleopeltis angusta & CHP, COL, DUR, GUA, GRO, JAL, MIC, NAY, OAX, PUE, VER & ** \\
\hline Polygala parryi & SLP & * \\
\hline Polygala subalata & COA, CMX, DUR, GUA, GRO, HID, JAL, MEX, MIC, NLE, OAX, PUE, QUE, SLP, VER & $* *$ \\
\hline Polypodium longipinnulatum & CHP, COL, GRO, HID, JAL, MIC, OAX, PUE, QUE, SLP, VER & * \\
\hline Polypodium sanctae-rosae & CHP, COL, GRO, HID, JAL, MEX, NAY y OAX & $* *$ \\
\hline Prochnyanthes mexicana & AGU, COL, DUR, GUA, JAL, MEX, MIC, MOR, NAY, QUE, ZAC & $* *$ \\
\hline Quercus grahamii & GRO, HID, JAL, MEX, MIC, OAX, PUE & * \\
\hline Roldana gonzaleziae & DUR, JAL, NAY, ZAC & $* *$ \\
\hline Roldana jurgensenii & CHP, OAX & * \\
\hline Rubus cymosus & CMX, GRO, JAL, MEX, MIC & * \\
\hline Schiedeella albovaginata & COL, CMX, JAL, MEX, MIC, MOR & * \\
\hline Scutellaria dumetorum & $\begin{array}{l}\text { CAM, CHP, COL, CMX, DUR, GUA, GRO, HID, JAL, MEX, MIC, MOR, NAY, NLE, } \\
\text { OAX, PUE, QUE, SLP, TAM, TLA, VER }\end{array}$ & $* *$ \\
\hline Stellaria cuspidata & $\begin{array}{l}\text { BCS, CHP, CHH, COA, COL, CDM, GUA, GRO, HID, JAL, MEX, MIC, MOR, NAY, } \\
\text { NLE, OAX, PUE, QRO, SLP, TAM, TLA, VER }\end{array}$ & $* *$ \\
\hline Thalictrum gibbosum & $\begin{array}{l}\text { AGU, CMX, DUR, GUA, GRO, HID,JAL, MEX, MIC, MOR, OAX, PUE, QRO, } \\
\text { SLP,TAM, TLA, VER }\end{array}$ & $* *$ \\
\hline Valeriana deltoidea & CHP, GRO, JAL, OAX & $*$ \\
\hline Verbesina cymbipalea & MEX & $*$ \\
\hline Yucca sp. $^{\mathrm{a}}$ & & $* *$ \\
\hline
\end{tabular}

tudio al tener valores más altos (492 especies, 285 géneros y 103 familias) contra lo reportado por Ponce-Vargas et al. (2006) para Lolotla, Hidalgo (359, 260, 103), López-Pérez et al. (2011) para Avándaro, Estado de México (391, 245, 98), Frías-Castro et al. (2013) para el Cerro El Tepopote, Jalisco $(370,214,76)$ y Guerrero-Hernández et al. (2014) para Juanacatlán, Jalisco $(290,195,80)$. Lo anterior a pesar de que el esfuerzo de muestreo fue mayor en algunos de esos trabajos; por ejemplo, los muestreos de Ponce-Vargas et al. (2006) cubrieron dos años, Frías-Castro et al. (2013) dos años y medio y López-Pérez et al. (2011) cinco años. En contraste, en el trabajo de Padilla-Velarde et al. (2008) para Arroyo Agua Fría, Colima, se obtienen valores superiores (716, 423, 127). Esto último se explica en gran medida debido a que en esa área se trabajó con seis tipos de vegetación (bosque de encino, bosque mesófilo de montaña, bosque de pino, bosque tropical subcaducifolio, vegetación secundaria, y ecotono entre bosque de encino y bosque mesófilo de montaña) lo que permite que se desarrollen condiciones propicias para que un número mayor de especies se presenten en el área. Además, esta área ya había sido objeto de varias colectas tal es el caso que el autor comenta que además de las colectas realizadas en el área se revisaron 3,500 ejemplares de herbario lo que aumenta considerablemente el esfuerzo de muestreo. La alta diversidad a nivel de especies, géneros y familias encontrada en el área de estudio, podría estar determinado por su ubicación, la convergencia de dos grandes zonas biogeográficas (Neártica y Neotropical; Rzedowski 1978), su amplio gradiente altitudinal y a la compleja topografía de la zona. Además de que el área es favorecida por la humedad proveniente del Pacífico, cerca de la mitad del área de estudio está conformada por una gran cañada (Figura 1) que actúa como un embudo por el que se desplaza la humedad hacia las porciones altas de la región.

Las familias que registraron mayor número de especies (Asteraceae, Fabaceae, Orchidaceae y Poaceae) coinciden con las cuatro familias más diversas de México (Villaseñor 2016); algo semejante ocurre en Sinaloa, solo que Orchidaceae desciende algunos lugares (Vega 2000). Este mismo patrón es el típico que se mantiene en otros trabajos florísticos en el país (Rzedowski 1996, Alcántara-Ayala \& Luna-Vega 2001, Ponce-Vargas et al. 2006, López-Pérez et al. 2011, 
Frías-Castro et al. 2013, Guerrero-Hernández et al. 2014, Morales-Saldaña et al. 2015, Morales-Arias et al. 2016, González-Elizondo et al. 2017, Macías-Rodríguez et al. 2018).

De manera semejante, los tres géneros más diversos (Quercus, Salvia y Muhlenbergia) son también de los más numerosos y ampliamente distribuidos en el país (Villaseñor 2016). Las especies de Quercus del área estudiada representan el 66.6-71.4\% de las especies reportadas para Sinaloa (Valencia-A 2004), las de Salvia el 36.6 \% (Martínez-Gordillo et al. 2017) y las de Muhlenbergia el $31.03 \%$ (Dávila et al. 2018). Además corresponden con los resultados de otros trabajos florísticos del occidente de México y con vegetación semejante (Frías-Castro et al. 2013, Guerrero-Hernández et al. 2014, Morales-Saldaña et al. 2015, Morales-Arias et al. 2016, González-Elizondo et al. 2017, Macías-Rodríguez et al. 2018). No es raro que en el ANP la mayor riqueza se concentre en estos géneros, ya que ocupan segundo, tercero y cuarto lugar respectivamente de los géneros más diversos en el norte de México (González-Elizondo et al. 2017).

Consideramos que el número de especies de Quercus es preliminar en virtud de la dificultad para su identificación. Diversos problemas como la gran variación morfológica de las especies (incluso dentro de un mismo individuo), descripciones originales deficientes o ambiguas, un número elevado de sinonimias, la frecuente hibridación, la falta de trabajos taxonómicos regionales y la diferencia de criterios de los especialistas (Spellenberg et al. 1998, Valencia-A 2004), dificultan la correcta identificación y por consiguiente determinar el número de especies presentes en un área. En consecuencia, en tales grupos de taxonomía complicada, además de la taxonomía tradicional, se recomienda emplear métodos moleculares como una fuente adicional de evidencia para esclarecer la identidad de los taxones. De tal manera que, en el inventario del Santuario se excluyeron 7 encinos a los que no fue posible asignarles un nombre específico. Por otra parte, se reconoce a $Q$. brachystachys y $Q$. reticulata como especies aceptadas debido a que los especímenes del área determinados bajo estos nombres se ajustan mejor a las descripciones y variación morfológica de tales taxones que aquellas de Q. crassifolia y Q. rugosa, donde respectivamente algunos autores las sumergen como sinónimos (Valencia-A 2004). Se acepta de manera provisional a ambas especies para dar constancia de que en el área de estudio existe tal constitución morfológica particular, aunque será necesario que se realice un estudio puntual para fundamentar el estatus taxonómico de ambas especies. En el listado se conservaron sólo 15 especies que correspondían con mayor claridad a las entidades descritas. La alta diversidad de encinos dentro del polígono inventariado es producto de un amplio gradiente altitudinal y de la mezcla de elementos de las zonas templadas y tropicales de la SMO (Spellenberg et al. 1998, González-Elizondo et al. 2007, 2012b).

Asimismo, el número de especies de encinos encontrados en el área de muestreo es similar a lo reportado por Frías-Castro et al. (2013) y Macías-Rodríguez et al. (2018), superior a lo de Padilla-Velarde et al. (2008), Guerrero-Hernández et al. (2014) y Morales-Saldaña et al. (2015), e inferior a lo de Harker et al. (2015) y Morales-Arias et al. (2016). Esto últi- mo posiblemente se deba a que el área de muestro es mayor en estos trabajos. Dado que la SMO ha sido sitio de diversificación de Quercus, y además de que se presenta la mayor diversidad de asociaciones de pinos, encinos y madroños a nivel mundial, no es extraño encontrar tal diversidad de encinos en la zona (Spellenberg et al. 1998, González-Elizondo et al. 2007, 2012a, 2012b).

Forma biológica de la flora. Las hierbas y los arbustos fueron las formas biológicas con mayor representación, lo que coincide con otros trabajos florísticos realizados en México (Rzedowski 1996, Alcántara-Ayala \& Luna-Vega 2001, Ponce-Vargas et al. 2006, López-Pérez et al. 2011, Frías-Castro et al. 2013, Guerrero-Hernández et al. 2014, Morales-Saldaña et al. 2015, Morales-Arias et al. 2016, Macías-Rodríguez et al. 2018). Que las hierbas concentren más especies no es inusual dado que son la forma biológica dominante en México (Villaseñor \& Ortiz 2014). Es posible que las extracciones de madera y el desmonte para agricultura en las áreas de amortiguamiento favorezcan este hábito al dejar espacios vacantes a hierbas pioneras (Martínez-De La Cruz et al. 2015). Por ejemplo, 37 especies de hierbas se localizaron sólo en sitios con algún grado de disturbio, en zonas desmontadas o a la orilla de caminos. La cantidad de plantas parásitas registradas (20) es mayor que las reportadas en trabajos florísticos similares, por ejemplo, 3 especies por Padilla-Velarde et al. (2008), 6 por Frías-Castro et al. (2013), 3 por Guerrero-Hernández et al. (2014), 8 por Harker et al. (2015) y 9 por Morales-Arias et al. (2016) y Macías-Rodríguez et al. (2018). En contraste el número de epífitas registradas fue menor que las reportadas en otros trabajos florísticos con vegetación similar (Alcántara-Ayala \& Luna-Vega 2001, Frías-Castro et al. 2013, Guerrero-Hernández et al. 2014, Harker et al. 2015, Durán-Fernández et al. 2016, Castillo-Hernández \& FloresOlvera 2017), dado que la colecta implica trepar árboles, solo se colectaron aquellas que se encontraban a una altura asequible, por lo que existe la posibilidad de que el número de plantas epífitas sea mayor.

Especies bajo categoría de riesgo. La presencia de 14 especies de plantas (Tabla 4) en alguna de las categorías de protección de la NOM-059-SEMARNAT-2010 (SEMARNAT 2010), o en la IUCN (2019), es una cifra alta comparada con las reportada en los estudios florísticos aquí cotejados. En la literatura analizada las especies dentro de alguna de las categorías que ameritan atención para la conservación varía de 3 (López-Pérez et al. 2011, Frías-Castro et al. 2013) a 16 (Padilla-Velarde et al. 2008). Por tanto, la relevancia del área en este aspecto de forma relativa puede categorizarse como moderada a alta. También destaca la presencia de 14 especies de aves bajo protección de acuerdo a López-Segoviano et al. (2019). Con base en esta información deberían promoverse estrategias integrales de conservación en el Santuario para garantizar la funcionalidad del ecosistema y la preservación de las especies.

Registros nuevos. El $10.77 \%$ de las plantas en el listado son registros nuevos para el estado de Sinaloa. Dado que 
el polígono del Santuario no representa ni $0.02 \%$ de la superficie de Sinaloa, y que se encuentra en un sitio muy próximo a una carretera de las más transitadas en el estado (carretera federal 40, Durango-Mazatlán), el porcentaje de registros nuevos es un indicador de la escasa exploración botánica en algunas de sus regiones. Con seguridad, muchos de los registros nuevos se encuentran también en otros sitios de Sinaloa pero no han sido aún documentados. Así mismo, el porcentaje de plantas por descubrirse debe ser considerable. También llama la atención que Lobelia macrocentron haya pasado desapercibida por 169 años a pesar de encontrarse cercana a la carretera Durango-Mazatlán, misma que ha sido un transecto de exploración botánica atractivo y visitado históricamente (Seemann 1852, McVaugh 1977, González-Elizondo et al. 2017, Ávila-González et al. 2018); sorprende también el hallazgo de una especie nueva de Bletia (Ávila-González et al. 2019). Al respecto, lo anterior denota la ineludible importancia de realizar inventarios biológicos como principal herramienta para el conocimiento y adecuado manejo de la biodiversidad.

\section{Agradecimientos}

Agradecemos por el apoyo financiero proporcionado para esta investigación al Consejo Nacional de Ciencia y Tecnología (CONACyT), al Programa Institucional de Formación de Investigadores (PIFI) del Instituto Politécnico Nacional, a Madrean Discovery Expeditions Scholarship y al Proyecto Inventario Florístico en La Reserva La Chara Pinta Sinaloa (SIP 20180521). Damos las gracias a Fernando Valdez, por brindar alojamiento en las cabañas de El Santuario durante las exploraciones botánicas, y a Santos-Vázquez por guiarnos y apoyarnos durante las mismas. Apreciamos el apoyo de Fernando Colin-Nolasco, Flor Isela Retana-Rentería, Norma Leticia Piedra-Leandro, Socorro González-Elizondo, Yolanda Herrera-Arrieta, por la valiosa ayuda en la identificación del material colectado. También se agradece Eduardo Contreras-Mora, Alberto Rivera y Raúl Narváez-Elizondo por la ayuda en el trabajo de campo.

\section{Literatura citada}

Ahlquist TK, Vincent MA. 2018. Trifolium sonorense (Fabaceae), a new species in the T. amabile species complex from Arizona and Mexico. Phytoneuron 1: 1-5.

Alcántara-Ayala O, Luna-Vega I. 2001. Análisis florístico de dos áreas con bosque mesófilo de montaña en el estado de Hidalgo, México: Eloxochitlán y Tlahuelompa. Acta Botanica Mexicana 54: 51-87.

DOI: https://doi.org/10.21829/abm54.2001.868

Anderson WR. 2014. Seven new species of neotropical Malpighiaceae. Acta Botanica Mexicana 109: 23-43.

DOI: https://doi.org/10.21829/abm109.2014.1146

APG (Angiosperm Phylogeny Group). 2016. An update of the Angiosperm Phylogeny Group classification for the orders and families of fowering plants: APG IV. Botanical Journal of the Linnean Society 181: 1-20.

DOI: https://doi.org/10.1111/boj.12385
Arriaga-Cabrera L, Espinoza-Rodríguez JM, Aguilar-Zúñiga C, Martínez-Romero E, Gómez-Mendoza L, Loa-Loza E. 2000. Regiones terrestres prioritarias de México. Escala de trabajo 1:1,000,000. México, DF: Comisión Nacional para el Conocimiento y uso de la Biodiversidad. ISBN: 970-9000-16-0

Ávila-González H, González-Gallegos JG, Rubio-Cardoza J, Castro-Castro A. 2018. The rediscovery of Lobelia macrocentron (Campanulaceae) after 169 years, with notes on the morphology, habitat and conservation status. Phytotaxa 374: 268-272. DOI: https://doi.org/10.11646/phytotaxa.374.3.9

Ávila-González H, González-Gallegos JG, Castro-Castro A, Rubio-Cardoza J. 2019. Bletia santosii (Orchidaceae), una especie nueva para Sinaloa, México. Brittonia.

DOI: https://doi.org/10.1007/s12228-019-09590-z

Beltrán J. 2003a. Bosque mesófilo de montaña. In: CifuentesLemus JL, Gaxiola-López J, eds. Atlas de los Ecosistemas de Sinaloa. Culiacán, Sinaloa: El Colegio de Sinaloa, pp. 303-308. ISBN: 9685439044, 9789685439046

Beltrán J. 2003b. Bosque de Quercus y Pinus de Sinaloa. In: Cifuentes L, Gaxiola L, eds. Atlas de los ecosistemas de Sinaloa. Culiacán: El Colegio de Sinaloa, pp. 297-302. ISBN: 968-5439-04-4

Borhidi A, Martínez-Salas E, Salas-Morales S. 2013. Estudios sobre rubiáceas mexicanas, $\mathrm{XL}$ tres nuevas especies del género Bouvardia Salisb. y la revalidación de una especie omisa de Centroamérica. Acta Botanica Hungarica 55: 1-16. DOI: https://doi.org/10.1556/ABot.55.2013.1-2.1

Castillo-Hernández LA, Flores-Olvera H. 2017. Floristic composition of the cloud forest of the Bicentenario Reserve, Zongolica, Veracruz, México. Botanical Sciences 95: 1-25. DOI: https://doi.org/10.17129/botsci.1223

Castro-Castro A, Zuno-Delgadillo O, Carrasco-Ortiz MA, Harker M, Rodríguez A. 2015. Novedades en el género Dahlia (Asteraceae: Coreopsideae) en Nueva Galicia, México. Botanical Sciences 93: 41-51.

DOI: http://dx.doi.org/10.17129/botsci.239

Christenhusz MJM, Reveal JL, Farjon A, Gardner MF, Mill RR, Chase MW. 2011. A new classification and linear sequence of extant gymnosperms. Phytotaxa 19: 55-70. DOI: https://doi.org/10.11646/phytotaxa.19.1.3

Cohen JI. 2018. A revision of the Mexican species of Lithospermum (Boraginaceae). Annals of the Missouri Botanical Garden 103: 200-257. DOI: https://doi.org/10.3417/2011067

CONABIO [Comisión Nacional para el Conocimiento y Uso de la Biodiversidad]. 2010. El bosque mesófilo de montaña en México: amenazas y oportunidades para su conservación y manejo sostenible. México, DF: Comisión Nacional para el Conocimiento y Uso de la Biodiversidad. ISBN: 978-6077607-35-9

CONANP [Comisión de nacional de áreas naturales protegidas]. 2008. Estudio Previo Justificativo para el establecimiento del área natural protegida con la categoría de Santuario "El Palmito”, en el estado de Sinaloa. México: Comisión Nacional de Áreas Naturales Protegidas http://biblioteca.semarnat. gob.mx/janium/Documentos/Ciga/Libros2013/CD003137a. pdf (accessed November 10, 2018)

Costea M, García-Ruíz I, Dockstader K, Stefanovic S. 2014. More problems despite bigger flowers: systematics of $\mathrm{Cus}$ - 
cuta tinctoria Clade (subgenus Grammica, Convolvulaceae) with description of six new species. Systematic Botany 38 : 1160-1187.

DOI: https://doi.org/10.1600/036364413x674887

Cruz-Durán R, Sousa SM. 2013. Eysenhardtia byei (Leguminosae, Papilionoideae), una especie nueva del Noroeste de México. Novon 22: 391-395.

DOI: https://doi.org/10.3417/2010117

Cruz-Durán R, Sousa SM. 2017. Cuatro especies nuevas de Indigofera (Leguminosae, Papilionoideae) para la Flora de México. Brittonia 69: 347-358.

DOI: https://doi.org/10.1007/s12228-017-9472-6

Dávila P, Mejia-Saulés MT, Soriano-Martínez AM, HerreraArrieta Y. 2018. Conocimiento taxonómico de la familia Poaceae en México. Botanical Sciences 96: 462-514. DOI: https://doi.org/10.17129/botsci.1894

Durán-Fernández A, Aguirre-Rivera JR, García-Pérez J, LevyTacher S, De Nova-Vázquez JA. 2016. Inventario florístico de la comunidad lacandona de Nahá, Chiapas, México. Botanical Sciences 94: 157-184.

DOI: https://doi.org/10.17129/botsci.248

Espejo-Serna A. 2014. Las plantas vasculares de los bosques mesófilos de montaña en México. In: Gual-Díaz, M, RendónCorrea A, eds. Bosques mesófilos de montaña de México: diversidad, ecología y manejo. México, D.F.: Comisión Nacional para el Conocimiento y Uso de la Biodiversidad, pp. 189-195. ISBN: 978-607-8328-07-9

Espinosa-Jiménez JA, Pérez-Farrera MA, Martínez-Camilo R. 2011. Inventario florístico del Parque Nacional Cañón del Sumidero, Chiapas, México. Boletín de la Sociedad Botánica de México 89: 37-82.

DOI: https://doi.org/10.17129/botsci.369

Estrada-C AE, Villarreal-Q J, López-E L. 2011. A new species of Dalea ser. Versicolores (Leguminosae: Amorpheae) from Durango, Mexico. Brittonia 63: 465-468.

DOI: https://doi.org/10.1007/s12228-011-9198-9

Estrada-Castillón AE, González-Elizondo MS, Villarreal-Quintanilla JA. 2016. A new species of Astragalus (Fabaceae, Faboideae) from Durango, Mexico. Phytotaxa 288: 91-95. DOI: http://dx.doi.org/10.11646/phytotaxa.288.1.10

Folk RA, Freudenstein JV. 2014. Revision of Heuchera section Rhodoheuchera subsections Hemsleyanae and Rosendahliae subsectio nova (Saxifragaceae). Systematic Botany 39: 850874. DOI: https://doi.org/10.1600/036364414X682300

Font-Quer P. 1953. Diccionario de Botánica. Barcelona: Labor. ISBN: 84-335-5804-8

Frías-Castro A, Castro-Castro A, González-Gallegos JG, Suárez-Muro EA, Rendón-Sandoval FJ. 2013. Flora vascular y vegetación del cerro El Tepopote, Jalisco, México. Botanical Sciences 91: 53-74.

DOI: http://dx.doi.org/10.17129/botsci.402

García-Martínez MA, Rodríguez A, McDonald HP. 2017. Validation of Calochortus ownbeyi (Liliaceae), a new species from northwestern Mexico. Phytotaxa 314: 241-250. DOI: https://doi.org/10.11646/phytotaxa.314.2.5

González-Elizondo MS, González-Elizondo M, Márquez-Linares MA. 2007. Vegetación y Ecorregiones de Durango. México, DF: Plaza y Valdez. ISBN: 9789709511703
González-Elizondo MS, González-Elizondo M, Sørensen PD. 2012a. Arbutus bicolor (Ericaceae, Arbuteae), a new species from Mexico. Acta Botanica Mexicana 99: 55-72. DOI: https://doi.org/10.21829/abm99.2012.19

González-Elizondo MS, González-Elizondo M. Tena-Flores J, Ruacho-González L, López-Enríquez I. 2012b. Vegetación de la Sierra Madre Occidental, México: una síntesis. Acta Botanica Mexicana 100: 351-403. DOI: https://doi.org/10.21829/abm100.2012.40

González-Elizondo MS, González-Elizondo M, López-Enríquez IL, Tena-Flores JA, González-Gallegos JG, RuachoGonzález L, Melgoza-Castillo A, Villarreal-Quintanilla AJ, Estrada-Castillón AE. 2017. Diagnóstico del conocimiento taxonómico y florístico de las plantas vasculares del norte de México. Botanical Sciences 95: 760-779.

DOI: http://dx.doi.org/10.17129/botsci.1865

González-Gallegos JG. 2013. Salvia albicalyx and Salvia topiensis (Lamiaceae), two new species from Durango, Mexico. Phytotaxa 77: 9-18.

DOI: http://dx.doi.org/10.11646/phytotaxa.77.1.3

González-Gallegos JG. 2016. Two new Salvia species (Lamiaceae) from the Sierra Madre Occidental, Durango, Mexico. Systematic Botany 40: 1093-1101.

DOI: https://doi.org/10.1600/036364415x690139

González-Gallegos JG, López-Enríquez IL. 2016. Salvia wixarika (Lamiaceae), a new species from Jalisco, Mexico, and novelties on Mexican Salvia with white corollas. Phytotaxa 260: $176-184$.

DOI: https://doi.org/10.11646/phytotaxa.260.2.7

González-Gallegos JG, López-Enríquez IL. 2017. Agastache sandersiana (Lamiaceae): a new species from northwestern Durango, Mexico. Journal of the Torrey Botanical Society 144: $97-103$.

DOI: https://doi.org/10.3159/TORREY-D-15-00057.1

González-Tamayo R, Hernández-Hernández L. 2010. Las orquídeas del occidente de México. Guadalajara: COECYTJAL. ISBN: 9786070040412

González-Rocha E, Castillo-Rivera MA, López-Ferrari AR, Espejo-Serna A. 2018. A multivariate analysis of the Pitcairnia palmeri group (Bromeliaceae: Pitcairnioideae). Phytotaxa 351: 219-228.

DOI: https://doi.org/10.11646/phytotaxa.351.3.3

Gual-Díaz M, Rendón-Correa A. eds. 2014. Bosques mesófilos de montaña de México: Diversidad, Ecología y Manejo. México DF: Comisión Nacional para el Conocimiento y Uso de la Biodiversidad. ISBN: 978-607-8328-07-9

Guerrero-Hernández R, González-Gallegos JG, Castro-Castro A. 2014. Análisis florístico de un bosque de Abies y el bosque mesófilo de montaña adyacente en Juanacatlán, Mascota, Jalisco, México. Botanical Sciences 92: 541-562.

DOI: http://dx.doi.org/10.17129/botsci.119

Gutiérrez-Ortega JS, Jiménez-Cedillo K, Pérez-Farrera MA, Martínez JF, Molina-Freaner F, Watano Y, Kajita T. 2018. Species definition of Dioon sonorense (Zamiaceae, Cycadales), and description of $D$. vovidesii, a new cycad species from northwestern Mexico. Phytotaxa 369: 107-114. DOI: http://doi.org/10.11646/phytotaxa.369.2.4

Gutiérrez-Sánchez RI, Castro-Castro A, González-Gallegos JG, 
López-Enríquez IL, Frías-Castro A. 2018. Synopsis of the spurred species of Lobelia section Stenotium (Campanulaceae) in Sierra Madre Occidental, Mexico, and the description of two new species. Phytotaxa 338: 33-48.

DOI: http://dx.doi.org/10.11646/phytotaxa.338.1.3

Harker M, Hernández-López L, Reynoso-Dueñas JJ, GonzálezVillarreal LM, Cedano-Maldonado M, Arias-García JA, Villaseñor-Ibarra L, Quintero-Fuentes V. 2015. Actualización de la flora vascular de San Sebastián del Oeste, Jalisco, México. Ibugana 8: 3-63.

Harris JG, Harris MW. 1994. Plant identification terminology. An illustrated glossary. Payson UT: Spring Lake Publishing. ISBN-10: 0964022168; ISBN-13: 978-0964022164

Henrickson J, Van Devender TR. 2010. A new Leucophyllum (Scrophulariaceae) from Sonora, Mexico. Journal of the Botanical Research Institute of Texas 4: 581-585.

Henrickson J, Fishbein M, Van Devender TR. 2011. Lepechinia yecorana (Lamiaceae), a new dioecious species from the Yecora area of Sonora, Mexico. Journal of the Botanical Research Institute of Texas 5: 67-74.

Hernández-Barón LJ, Cerros-Tlatilpa R, Espejo-Serna A, González-Elizondo M, López-Ferrari AR. 2018. A new species of Amyris (Rutaceae) from Durango, Mexico. Systematic Botany 43: 801-805.

DOI: https://doi.org/10.1600/036364418x697517

IPNI. 2019. International Plant Name Index https://www.ipni. org/ (accessed March 1-30, 2019)

IUCN [Unión Internacional para la Conservación de la Naturaleza] 2019. The IUCN Red List of Threatened Species. https://www.iucnredlist.org (accessed March 30, 2019)

Jimeno-Sevilla HD, Carrillo-Reyes P. 2010. Echeveria perezcalixii (Crassulaceae), una especie nueva del occidente de México. Brittonia 62: 303-308.

DOI: https://doi.org/10.1007/s12228-010-9137-1

López-Pérez Y, Tejero-Díez JD, Torres-Díaz AN, Luna-Vega I. 2011. Flora del bosque mesófilo de montaña y vegetación adyacente en Avándaro, Valle de Bravo, estado de México, México. Boletín de la Sociedad Botánica de México 88: 3553. DOI: http://dx.doi.org/10.17129/botsci.304

López-Segoviano G, Díaz-Verduzco L, Arenas-Navarro M, Arizmendi M. 2019. Diversidad estacional de aves en una región prioritaria para la conservación en el centro oeste de la Sierra Madre Occidental. Revista Mexicana de Biodiversidad 90: 1-16.

DOI: https://doi.org/10.22201/ib.20078706e.2019.90.2754

Lorence DH, Van Devender TR, Ferguson GM. 2018. Chiococca grandiflora (Rubiaceae), a new species from Northern Mexico. PhytoKeys 98: 73-83.

DOI: http://dx.doi.org/10.3897/phytokeys.98.25170

Lot A, Chiang F. 1986. Manual de Herbario. Administración, Manejo de Colecciones, Técnicas de Recolección y Preparación de Ejemplares Botánicos. México, DF: Consejo Nacional de la Flora de México, AC. ISBN: 968-6144-00-5

Luna-Vega I, Ocegueda-Cruz S, Alcántara-Ayala O. 1994. Florística y notas biogeográficas del bosque mesófilo de montaña del municipio de Tlanchinol, Hidalgo, México. Anales del Instituto de Biología, Serie Botánica 65: 31-62.

Macías-Rodríguez MA, Frías-Ureña HG, Contreras-Rodríguez
SH, Frías-Castro A. 2018. Vascular plants and vegetation of the Sayula sub-basin, Jalisco, Mexico. Botanical Sciences 96: 103-137. DOI: https://doi.org/10.17129/botsci.1030

Márquez-Linares M, González-Elizondo MS. 1998. Composición y estructura del estrato arbóreo de un bosque de pinoencino en Durango, México. Agrociencia 32: 413-419.

Martínez-De La Cruz I, Vibrans H, Lozada-Pérez L, Romero-Manzanares A, Aguilera-Gómez LI, Rivas-Manzano IV. 2015. Plantas ruderales del área urbana de Malinalco, estado de México. Botanical Sciences 93: 907-919.

DOI: https://dx.doi.org/10.17129/botsci.213

Martínez-Gordillo MJ, Bedolla-García B, Cornejo-Tenorio G, Fragoso-Martínez I, García-Peña MR, González-Gallegos JG, Lara-Cabrera SI, Zamudio S. 2017. Lamiaceae de México. Botanical Sciences 95: 780-806.

DOI: http://dx.doi.org/10.17129/botsci.1871

Mayfield MH, Steinmann VW. 2010. Euphorbia spellenbergiana (Euphorbiaceae), a new species from Mexico. Acta Botanica Mexicana 90: 43-50.

DOI: https://doi.org/10.21829/abm90.2010.298

Mcdonnell A, Fishbein M. 2016. Polystemma canisferum (Apocynaceae, Asclepiadoideae): a distinctive new gonoloboid milkweed vine from Sonora, Mexico. Phytotaxa 246: 078084. DOI: http://dx.doi.org/10.11646/phytotaxa.246.1.6

McVaugh R. 1977. Edward Palmer, plant explorer of the American West. Norman: University of Oklahoma Press. ISBN: 978-091-37-2826-0

Mickel JT, Smith AR. 2004. The Pteridophytes of Mexico. New York: Memoirs of the New York Botanical Garden 88: 11054.

Morales-Arias JG, Cuevas-Guzmán R, Rodríguez-Hernández JL, Guzmán-Hernández L, Núñez-López NM, Sánchez-Rodríguez EV, Solís-Magallanes A, Santana-Michel FJ. 2016. Flora vascular de Villas de Cacoma, Sierra de Cacoma, Jalisco, México. Botanical Sciences 94: 393-418.

DOI: http://dx.doi.org/10.17129/botsci.447

Morales-Saldaña S, Martínez-Ambriz E, Valencia-Á S. 2015. Estudio florístico y de la vegetación del municipio de Buenavista de Cuéllar, Guerrero, México. Botanical Sciences 93: 73-95. DOI: http://dx.doi.org/10.17129/botsci.234

Moreno NP. 1984. Glosario Botánico Ilustrado. Xalapa: Instituto Nacional de Investigaciones sobre Recursos Bióticos (CECSA). ISBN: 968-26-04-34-6

Naturalista. 2018. Comisión Nacional para el Conocimiento y Uso de la Biodiversidad (CONABIO - Naturalista). < http:// www.naturalista. $\mathrm{mx}>$ (accessed November 8-30, 2018, March 1-30, 2019)

Nesom GL. 2010. A new species of Erigeron (Asteraceae: Astereae) from northwestern Chihuahua, Mexico. Phytoneuron 28: $1-6$.

Nesom GL. 2014. Taxonomy of Erythranthe sect. Erythranthe (Phrymaceae). Phytoneuron 31: 1-41.

Nesom GL. 2017. Erythranthe diminuens (Phrymaceae), a new species of sect. Simiolus from Sonora. Phytoneuron 7: 1-5.

Nesom GL, 2018. New species of Baccharis (Asteraceae) from western Mexico. Phytoneuron 28: 1-20.

Padilla-Velarde E, Cuevas-Guzmán R, Koch SD. 2008. Plantas vasculares y vegetación de la parte alta del Arroyo Agua Fría, 
municipio de Minatitlán, Colima, México. Acta Botanica Mexicana 84: 25-72.

DOI: https://doi.org/10.21829/abm84.2008.1066

Pérez-Cálix E. 2016. Sedum pyriseminum (Crassulaceae), a morphologically remarkable new annual species from the state of Durango, Mexico. Phytotaxa 255: 297-300.

DOI: http://dx.doi.org/10.11646/phytotaxa.255.3.13

Ponce-Vargas A, Luna-Vega AI, Alcántara-Ayala O, Ruiz-Jiménez CA. 2006. Florística del bosque mesófilo de montaña de Monte Grande, Lolotla, Hidalgo, México. Revista Mexicana de Biodiversidad 77: 177-190.

DOI: http://dx.doi.org/10.22201/ib.20078706e.2006.002.333

Ramírez-Noya D, González-Elizondo MS, Molina-Torres J. 2011. Heliopsis suffruticosa (Compositae, Heliantheae), una nueva especie del occidente de Zacatecas. Acta Botanica Mexicana 97: 39-47.

DOI: https://doi.org/10.21829/abm97.2011.248

Reyes-Santiago J, González-Zorzano O, Kristen M. 2012a. Echeveria juliana (Crassulaceae), a new species from Sinaloa, Mexico. Haseltonia 18: 52-55.

DOI: https://doi.org/10.2985/026.018.0107

Reyes-Santiago J, González-Zorzano O, Etter J. 2012b. Sedum kristenii (Crassulaceae), A new species from Durango, Mexico. Haseltonia 18: 48-51.

DOI: https://doi.org/10.2985/026.018.0106

Reyes-Santiago J, Etter L, Kristen M. 2015. Sedum piaxtlaense (crassulaceae), a new species from Durango, México. Haseltonia 20: 58-63. DOI: https://doi.org/10.2985/026.020.0110

Reyes-Santiago J, Etter J, Kristen M. 2017. Sedum sinforosanum (Crassulaceae), a new species from the State of Chihuahua, Mexico. Cactus and Succulent Journal 89: 166-170. DOI: https://doi.org/10.2985/015.089.0404

Rodríguez A, Ortiz-Catedral L. 2013. Echeandia novogaliciana and E. crudeniana (Anthericaceae): two new species from western Mexico. Brittonia 65: 345-350.

DOI: https://doi.org/10.1007/s12228-012-9293-6

Ruiz-Sánchez E, Castro-Castro A. 2016. Otatea nayeeri (Poaceae: Bambusoideae: Bambuseae: Guaduinae), a new species endemic to Nayarit, Mexico. Phytotaxa 267: 211218. DOI: http://dx.doi.org/10.11646/phytotaxa.267.3.4

Ruiz-Sánchez E, Castro-Castro A, Clark LG. 2017. Chusquea septentrionalis sp. nov. (Poaceae: Bambusoideae) from the Madrean Region in Durango, Mexico. Nordic Journal of Botany 35: 546-551. DOI: https://doi.org/10.1111/njb.01606

Rzedowski J. 1978. Vegetación de México. México, DF: Limusa. ISBN: 968-18-0002-8

Rzedowski J. 1996. Análisis preliminar de la flora vascular de los bosques mesófilos de montaña de México. Acta Botanica Mexicana 35: 25-44.

DOI: https://doi.org/10.21829/abm35.1996.955

Rzedowski J. 2016. Ocho especies nuevas de Lobelia (Campanulaceae) de México. Phytoneuron 67: 1-18.

Saarela JM, Peterson PM, González MS, Rosen DJ. 2010. Eleocharis cryptica (Cyperaceae), a dwarf new species from Durango, Mexico. Brittonia 62: 233-238.

DOI: https://doi.org/10.1007/s12228-009-9111-y

Sánchez-Ken JG. 2010. Two new species of Paspalum (Paniceae: Panicoideae: Poaceae), a preliminary checklist of the genus in Mexico, and the identity of P. crinitum. Revista Mexicana de Biodiversidad 81: 629-647.

DOI: http://dx.doi.org/10.22201/ib.20078706e.2010.003.636

Scheinvar L, Olalde-Parra G, Gallegos-Vazquez C, MoralesSandoval J. 2018. A new species of Opuntia (Cactaceae) from coniferous and Quercus forest of northern Mexico. Bradleya 36: 5-12. DOI: https://doi.org/10.25223/brad.n36.2018.a4

Seemann B. 1852. The botany of the voyage of H. M. S. Herald, under the command of Captain Henry Kellett, R.N., C.B., during the years 1845-51. Londres: Lovell Reeve.

SEINet. 2018. Arizona - New Mexico Chapter. http://swbiodiversity.org/seinet/collections/harvestparams.php (accessed November 8-30, 2018 - March 1-30, 2019)

SEMARNAT [Secretaría del Medio Ambiente y Recursos Naturales]. 2008. AVISO por el que se informa al público en general que están a su disposición los estudios realizados para justificar la expedición del Decreto por el que se pretende declarar como área natural protegida con el carácter de Santuario, la zona conocida como Ejido El Palmito con una superficie de 1,150-00-00 hectáreas, localizada en el Municipio de Concordia, en el estado de Sinaloa, promovida por el Ejido El Palmito. Diario oficial de la federación. 2da Sección, 5 de Junio de 2008.

SEMARNAT [Secretaría del Medio Ambiente y Recursos Naturales]. 2010. Norma Oficial Mexicana NOM-059-SEMARNAT-2010, Protección ambiental - Especies nativas de México de flora y fauna silvestres - Categorías de riesgo y especificaciones para su inclusión, exclusión o cambio - Lista de especies en riesgo. Diario Oficial de la Federación. 2da Sección, 30 de diciembre de 2010.

Semple JC. 2017. Solidago spellenbergii (Asteraceae: Astereae), a new species of goldenrod from Mexico. Phytoneuron 75: $1-8$.

Spellenberg R. 2014. Quercus barrancana (sect. Quercus, white oaks), a new species from northwestern Mexico. Phytoneuron 105: 1-12.

Spellenberg R, Bacon JF, González-Elizondo MS. 1998. Los encinos (Quercus, Fagaceae) en un transecto sobre la Sierra Madre Occidental. Boletín del Instituto de Botánica 5: 357-387.

Steinmann VW. 2014. Croton lindquistii (Euphorbiaceae): a new arborescent species from western Mexico. Phytotaxa 166: 235-240. DOI: http://dx.doi.org/10.11646/phytotaxa.166.3.4

Tropicos. 2018-2019. Missouri Botanical Garden http://www. tropicos.org (accessed November 8-30, 2018, March 1-30, 2019)

Turner BL. 2011. Brickellia enigmatica (Asteraceae: Eupatorieae), a new species from north-central Mexico. Phytologia 93: 322-329.

Turner BL. 2012. Recension of the mexican species of Zaluzania (Asteraceae: Heliantheae). Phytologia 94: 319-333.

Turner BL. 2013a. A new species of Adenophyllum (Asteraceae: Tageteae) from northwestern Mexico. Phytologia 95: 18-20.

Turner BL. 2013b. Two novel Stevias (Asteraeae: Eupatorieae) from North-Western Mexico. Phytologia 95: 250-251.

Turner BL. 2013c. Stevia reinana (Asteraceae: Eupatorieae), a new species from near Yécora, Sonora, Mexico. Phytologia 95: 233-34. 
Turner BL. 2015. Three new species of Stevia (Asteraceae: Eupatorieae) from northern Mexico. Phytologia 97: 25-31.

Van Devender TR, Nesom GL. 2012. Glandularia malpaisana (Verbenaceae), a new species from Sonora, Mexico. Phytoneuron 65: 1-6.

Valencia-A S. 2004. Diversidad del género Quercus (Fagaceae) en México. Boletín de la Sociedad Botánica de México 75: 33-53. DOI: https://doi.org/10.17129/botsci.1692

Vega R. 2000. Catálogo y base de datos preliminar de la flora de Sinaloa. Universidad Autónoma de Sinaloa. Facultad de Agronomía. Informe final SNIB-CONABIO proyecto No. L057. México, DF http://www.conabio.gob.mx/institucion/ proyectos/resultados/InfL057.pdf (accessed April 20, 2019)

Villarreal-Quintanilla JA, Estrada-Castillón AE, Jasso de Rodríguez D. 2009. El género Gentiana (Gentianaceae) en México. Polibotánica 27: 1-16.

Villaseñor JL. 2010. El Bosque Húmedo de Montaña en México y sus Plantas Vasculares: Catálogo Florístico-Taxonómico. México, DF.: Comisión Nacional para el Conocimiento y Uso de la Biodiversidad/Universidad Nacional Autónoma de México. ISBN: 978-607-02-1557-5

Editor de sección: Martha González-Elizondo

Contribución de los autores: HÁG (https://orcid.org/0000-00034110-8846), JGG (https://orcid.org/0000-0003-3610-9086), ILLE (https://orcid.org/0000-0003-0075-5692) y LRG (https://orcid. org/0000-0001-5776-4237) participaron en el diseño de la propuesta. ACC (https://orcid.org/0000-0002-2864-5180), HÁG, JGG y JRC realizaron los muestreos. ACC, HÁG y JGGG realizaron la identificación de los ejemplares. HÁG, ILLE y LRG trabajaron en el procesamiento y análisis de datos. LRG elaboró los mapas. HÁG escribió el documento y JGGG realizó la corrección. Todos los autores leyeron, revisaron y aprobaron el manuscrito.
Villaseñor JL, Gual-Díaz, M. 2014. El bosque mesófilo de montaña en México y sus plantas con flores. In: Gual-Díaz, M, Rendón-Correa A. eds. Bosques Mesófilos de Montaña de México: Diversidad, Ecología y Manejo. México, DF: Comisión Nacional para el Conocimiento y Uso de la Biodiversidad, pp. 221-235. ISBN: 978-607-8328-07-9

Villaseñor JL, Ortiz E. 2014. Biodiversidad de las plantas con flores (División Magnoliophyta) en México. Revista Mexicana de Biodiversidad 85: 134-142.

DOI: https://doi.org/10.7550/rmb.31987

Villaseñor J. 2016. Checklist of the native vascular plants of Mexico. Revista Mexicana de Biodiversidad 87: 559-902. DOI: https://doi.org/10.1016/j.rmb.2016.06.017

Wallnofer B. 2015. A new species and two new synonyms of Diospyros (Ebenaceae) from Mexico. Stapfia 103: 111-113.

Zamudio S, Juárez-Gutiérrez HD, Hernández-Rendón J. 2018. Cuatro especies nuevas de Pinguicula (Lentibulariaceae) de México. Phytoneuron 14: 1-20. 
Apéndice 1. Listado de la flora vascular del Santuario El Palmito (La Chara Pinta), Sinaloa, México.

Colectores (Cols.): AAR (Anton A. Reznicek), ACC (Arturo Castro-Castro), ACS (Andrew C. Sanders), CPD (Cecilia Pulido-Díaz), DJP (Donal J. Pinkava), FCN (Luis Fernando Colin-Nolasco), HAG (Heriberto Ávila-González), JCR (Jaciel Rubio Cardoza), JGG (Jesús Guadalupe González-Gallegos), KR (Karen Liliana Rostro del Muro), L (Lehto), LL (Irma Lorena López-Enríquez), Nat (Naturalista), PVR (Perla Velázquez-Ríos), RDW (Richard D. Worthington), RR (Rocío Rivas), RVA (Rito Vega Aviña) y ZLD (Zazil Gabriela Lopez-Dellamary Tello). Forma biológica: Hábito (A): a (árbol), ar (arbusto), b (bejuco o liana), g (globosa), h (hierba), r (rosetófila). Hábitat (H): E (epífito), R (rupícola), T (terrestre). Tipo de nutrición (N): A (autótrofa), M (hemiparásita), O (holoparásita). Duración del ciclo de vida (C): A (anual) P (perenne). Símbolos: especie nueva $(*)$, endémica del area $(\bullet)$, Registros nuevos para Sinaloa $(\dagger)$.

\begin{tabular}{|c|c|c|c|c|c|}
\hline & Cols./ $\mathbf{N}^{\circ}$ de colecta & $\mathbf{A}$ & $\mathbf{H}$ & $\mathbf{N}$ & $\mathbf{C}$ \\
\hline \multicolumn{6}{|l|}{ L I C O F I T A S } \\
\hline \multicolumn{6}{|l|}{ ISOETACEAE } \\
\hline Isoetes montezumae A.A. Eaton & AAR-11118 & $\mathrm{h}$ & $\mathrm{T}$ & A & $\mathrm{P}$ \\
\hline \multicolumn{6}{|l|}{ SELAGINELLACEAE } \\
\hline Selaginella pallescens (C. Presl) Spring & HAG-512 & $\mathrm{h}$ & $\mathrm{R}$ & A & $\mathrm{P}$ \\
\hline \multicolumn{6}{|l|}{ HELECHOS } \\
\hline \multicolumn{6}{|l|}{ ASPLENIACEAE } \\
\hline Asplenium castaneum Schltdl. \& Cham. $\uparrow$ & HAG-185 & $\mathrm{h}$ & $\mathrm{T}, \mathrm{E}, \mathrm{R}$ & A & $\mathrm{P}$ \\
\hline Asplenium cuspidatum Lam. & HAG-400a & $\mathrm{h}$ & $\mathrm{R}, \mathrm{E}, \mathrm{T}$ & A & $\mathrm{P}$ \\
\hline Asplenium monanthes $\mathrm{L}$. & DJP-12886 & $\mathrm{h}$ & $\mathrm{T}, \mathrm{R}$ & A & $\mathrm{P}$ \\
\hline Asplenium sessilifolium Desv. & HAG-374 & $\mathrm{h}$ & $\mathrm{T}, \mathrm{R}$ & A & $\mathrm{P}$ \\
\hline \multicolumn{6}{|l|}{ BLECHNACEAE } \\
\hline Blechnum occidentale L. $\uparrow$ & HAG-479 & $\mathrm{h}$ & $\mathrm{R}$ & A & $\mathrm{P}$ \\
\hline Woodwardia spinulosa M. Martens \& Galeotti & HAG-271 & $\mathrm{h}$ & $\mathrm{T}$ & A & $\mathrm{P}$ \\
\hline \multicolumn{6}{|l|}{ DENNSTAEDTIACEA } \\
\hline Pteridium aquilinum (L.) Kuhn & HAG-54 & $\mathrm{h}$ & $\mathrm{T}$ & A & $\mathrm{P}$ \\
\hline \multicolumn{6}{|l|}{ DRYOPTERIDACEAE } \\
\hline Dryopteris cinnamomea (Cav.) C. Chr. & RVA-3025 & $\mathrm{h}$ & $\mathrm{T}$ & A & $\mathrm{P}$ \\
\hline Elaphoglossum erinaceum (Fée) T. Moore var. occidentale Mickel & HAG-184 & $\mathrm{h}$ & $\mathrm{R}, \mathrm{E}$ & A & $\mathrm{P}$ \\
\hline Elaphoglossum gratum (Fée) T. Moore & RVA-3126 & $\mathrm{h}$ & $E, R$ & A & $\mathrm{P}$ \\
\hline Elaphoglossum mulleri (E. Fourn.) C. Chr. & LFC-1265 & $\mathrm{h}$ & $\mathrm{E}, \mathrm{R}$ & A & $\mathrm{P}$ \\
\hline Elaphoglossum petiolatum (Sw.) Urb. & HAG-204 & $\mathrm{h}$ & $\mathrm{E}$ & A & $\mathrm{P}$ \\
\hline Elaphoglossum piloselloides (C. Presl) T. Moore & HAG-792 & $\mathrm{h}$ & $\mathrm{R}$ & A & $\mathrm{P}$ \\
\hline Phanerophlebia nobilis (Schltdl. \& Cham.) C. Presl. & HAG-468 & $\mathrm{h}$ & $\mathrm{T}$ & A & $\mathrm{P}$ \\
\hline \multicolumn{6}{|l|}{ OPHIOGLOSSACEAE } \\
\hline Botrychium virginianum (L.) Sw. & HAG-501 & $\mathrm{h}$ & $\mathrm{T}$ & A & $\mathrm{P}$ \\
\hline Ophioglossum crotalophoroides Walter & HAG-791 & $\mathrm{h}$ & $\mathrm{T}$ & A & $\mathrm{P}$ \\
\hline \multicolumn{6}{|l|}{ PLAGIOGYRIACEAE } \\
\hline Plagiogyria pectinata (Liebm.) Lellinger & HAG-203 & $\mathrm{h}$ & $\mathrm{T}$ & A & $\mathrm{P}$ \\
\hline \multicolumn{6}{|l|}{ POLYPODIACEAE } \\
\hline Campyloneurum angustifolium (Sw.) Fée & HAG-182 & $\mathrm{h}$ & $\mathrm{E}$ & A & $\mathrm{P}$ \\
\hline Pleopeltis angusta Humb. \& Bonpl. ex Willd. $\dagger$ & HAG-84 & $\mathrm{h}$ & $E, R$ & A & $\mathrm{P}$ \\
\hline Pleopeltis macrocarpa (Bory ex Willd.) Kaulf. & RVA-3024 & $\mathrm{h}$ & $\mathrm{E}$ & A & $\mathrm{P}$ \\
\hline Pleopeltis madrensis (J. Sm.) A. R. Sm. \& Tejero & HAG-456 & $\mathrm{h}$ & $\mathrm{R}$ & A & $\mathrm{P}$ \\
\hline Pleopeltis polylepis (Roem. ex Kunze) T. Moore & PVR-82 & $\mathrm{h}$ & $\mathrm{E}$ & A & $\mathrm{P}$ \\
\hline Polypodium longipinnulatum E. Fourn. $\dagger$ & HAG-467 & $\mathrm{h}$ & $\mathrm{E}$ & A & $\mathrm{P}$ \\
\hline
\end{tabular}




\begin{tabular}{|c|c|c|c|c|c|}
\hline & Cols./No de colecta & $\mathbf{A}$ & H & $\mathbf{N}$ & $\mathbf{C}$ \\
\hline Polypodium polypodioides (L.) Watt & PVR-83 & $\mathrm{h}$ & $\mathrm{E}$ & A & $\mathrm{P}$ \\
\hline Polypodium sanctae-rosae (Maxon) C. Chr. $\dagger$ & HAG-55 & $\mathrm{h}$ & $\mathrm{R}$ & A & $\mathrm{P}$ \\
\hline Polypodium subpetiolatum Hook. & HAG-56 & $\mathrm{h}$ & $\mathrm{R}$ & A & $\mathrm{P}$ \\
\hline Thelypteris rudis (Kunze) Proctor & HAG-469 & $\mathrm{h}$ & $\mathrm{T}$ & A & $\mathrm{P}$ \\
\hline \multicolumn{6}{|l|}{ PTERIDACEAE } \\
\hline Adiantum andicola Liebm. & HAG-223 & $\mathrm{h}$ & $\mathrm{T}, \mathrm{R}$ & A & $\mathrm{P}$ \\
\hline Adiantum poiretii Wikstr. & HAG-246 & $\mathrm{h}$ & $\mathrm{T}$ & A & $\mathrm{P}$ \\
\hline Cheilanthes farinosa (Forssk.) Kaulf. & HAG-452 & $\mathrm{h}$ & $\mathrm{R}$ & A & $\mathrm{P}$ \\
\hline Gaga hirsuta (Link) F. W. Li \& Windham & HAG-58 & $\mathrm{h}$ & $\mathrm{T}, \mathrm{R}$ & A & $\mathrm{P}$ \\
\hline Myriopteris lendigera (Cav.) J. Sm. & FCN-1262 & $\mathrm{h}$ & $\mathrm{R}$ & A & $\mathrm{P}$ \\
\hline Pellaea ternifolia (Cav.) Link & HAG-455 & $\mathrm{h}$ & $\mathrm{R}$ & A & $\mathrm{P}$ \\
\hline \multicolumn{6}{|l|}{ WOODSIACEAE } \\
\hline Woodsia mollis (Kaulf.) J. Sm. & HAG-572 & $\mathrm{h}$ & $\mathrm{T}$ & A & $\mathrm{P}$ \\
\hline \multicolumn{6}{|l|}{ GIMN O S P E R M A S } \\
\hline \multicolumn{6}{|l|}{ CUPRESSACEAE } \\
\hline Juniperus poblana (Martínez) R. P. Adams var. decurrens R. P. Adams & HAG-295 & a, ar & $\mathrm{T}$ & A & $\mathrm{P}$ \\
\hline Juniperus sp. & HAG-241 & a & $\mathrm{T}$ & A & $\mathrm{P}$ \\
\hline \multicolumn{6}{|l|}{ PINACEAE } \\
\hline Abies neodurangensis Debreczy, Rácz \& S. Salazar & HAG-309 & a & $\mathrm{T}$ & A & $P$ \\
\hline Pinus douglasiana Martínez & JRC-17 & $\mathrm{a}$ & $\mathrm{T}$ & A & $\mathrm{P}$ \\
\hline Pinus durangensis Martínez & HAG-65 & $\mathrm{a}$ & $\mathrm{T}$ & A & $P$ \\
\hline Pinus herrerae Martínez & HAG-341 & $\mathrm{a}$ & $\mathrm{T}$ & A & $\mathrm{P}$ \\
\hline Pinus lumholtzii B. L. Rob. \& Fernald & JRC-11 & $\mathrm{a}$ & $\mathrm{T}$ & A & $\mathrm{P}$ \\
\hline Pinus maximinoi $\mathrm{H}$. E. Moore & HAG-101 & $\mathrm{a}$ & $\mathrm{T}$ & A & $\mathrm{P}$ \\
\hline Pinus strobiformis Engelm. & HAG-261 & $\mathrm{a}$ & $\mathrm{T}$ & A & $\mathrm{P}$ \\
\hline \multicolumn{6}{|l|}{ A N G I OSPERM A S } \\
\hline \multicolumn{6}{|l|}{ MAGNOLÍDEAS } \\
\hline \multicolumn{6}{|l|}{ LAURACEAE } \\
\hline Litsea glaucescens Kunth & HAG-358 & ar & $\mathrm{T}$ & A & $\mathrm{P}$ \\
\hline \multicolumn{6}{|l|}{ MAGNOLIACEAE } \\
\hline Magnolia tarahumara (A. Vázquez) A. Vázquez & HAG-311 & $\mathrm{a}$ & $\mathrm{T}$ & A & $\mathrm{P}$ \\
\hline \multicolumn{6}{|l|}{ PIPERACEAE } \\
\hline Peperomia campylotropa A. W. Hill. & HAG-585 & $\mathrm{h}$ & $\mathrm{R}$ & A & $\mathrm{P}$ \\
\hline Peperomia galioides Kunth & HAG-214 & $\mathrm{h}$ & $\mathrm{R}, \mathrm{E}$ & A & $\mathrm{P}$ \\
\hline \multicolumn{6}{|l|}{ MONOCOTILEDÓNEAS } \\
\hline \multicolumn{6}{|l|}{ ALSTROEMERIACEAE } \\
\hline Bomarea hirtella (Kunth) Herb. & HAG-22 & $\mathrm{b}$ & $\mathrm{T}$ & A & $\mathrm{P}$ \\
\hline \multicolumn{6}{|l|}{ AMARYLLIDACEAE } \\
\hline Allium glandulosum Link \& Otto & HAG-135 & $\mathrm{h}$ & $\mathrm{T}$ & A & $\mathrm{P}$ \\
\hline Sprekelia formosissima (L.) Herb. & Nat. & $\mathrm{h}$ & $\mathrm{T}$ & A & $\mathrm{P}$ \\
\hline \multicolumn{6}{|l|}{ ARACEAE } \\
\hline Arisaema macrospathum Benth. & HAG-463 & $\mathrm{h}$ & $\mathrm{T}$ & A & $\mathrm{P}$ \\
\hline \multicolumn{6}{|l|}{ ARECACEAE } \\
\hline Chamaedorea pochutlensis Liebm. & HAG-470 & ar & $\mathrm{T}$ & A & $\mathrm{P}$ \\
\hline
\end{tabular}




\begin{tabular}{|c|c|c|c|c|c|}
\hline & Cols. $/ \mathbf{N}^{\circ}$ de colecta & $\mathbf{A}$ & $\mathbf{H}$ & $\mathbf{N}$ & $\mathbf{C}$ \\
\hline \multicolumn{6}{|l|}{ ASPARAGACEAE } \\
\hline Agave attenuata Salm-Dyck & HAG-304 & $\mathrm{r}$ & $\mathrm{R}, \mathrm{E}$ & A & $\mathrm{P}$ \\
\hline Agave maximiliana Baker & HAG-129 & $\mathrm{r}$ & $\mathrm{T}$ & A & $\mathrm{P}$ \\
\hline Agave schidigera Lem. & HAG-305 & $\mathrm{r}$ & $\mathrm{T}, \mathrm{R}$ & A & $\mathrm{P}$ \\
\hline Echeandia longipedicellata Cruden & HAG-12 & $\mathrm{h}$ & $\mathrm{T}$ & A & $\mathrm{P}$ \\
\hline Manfreda singuliflora (S. Watson) Rose & HAG-580 & $\mathrm{h}$ & $\mathrm{T}$ & A & $\mathrm{P}$ \\
\hline Milla biflora Cav. & HAG-108 & $\mathrm{h}$ & $\mathrm{T}$ & A & $\mathrm{P}$ \\
\hline Nolina sp. & HAG-222 & $\mathrm{r}$ & $\mathrm{T}$ & A & $\mathrm{P}$ \\
\hline Prochnyanthes mexicana (Zucc.) Rose $\dagger$ & ACC-4531 & $\mathrm{h}$ & $\mathrm{T}$ & A & $\mathrm{P}$ \\
\hline Yucca sp. $\dagger$ & ACC- -4535 & $\mathrm{r}$ & $\mathrm{T}$ & A & $\mathrm{P}$ \\
\hline \multicolumn{6}{|l|}{ BROMELIACEAE } \\
\hline Pitcairnia robert-downsii González-Rocha, Espejo, López-Ferr. \& M. Castillo † & HAG-413 & $\mathrm{h}$ & $\mathrm{R}$ & A & $\mathrm{P}$ \\
\hline Tillandsia borealis López-Ferr. \& Espejo & HAG-130 & $\mathrm{h}$ & $\mathrm{E}$ & A & $\mathrm{P}$ \\
\hline Tillandsia bourgaei Baker & KR-9 & $\mathrm{h}$ & $\mathrm{E}$ & A & $\mathrm{P}$ \\
\hline Tillandsia macdougallii L.B. Sm. & HAG-370 & $\mathrm{h}$ & $\mathrm{E}$ & A & $\mathrm{P}$ \\
\hline \multicolumn{6}{|l|}{ COMMELINACEAE } \\
\hline Commelina tuberosa $\mathrm{L}$. & HAG-547 & $\mathrm{h}$ & $\mathrm{T}$ & A & $\mathrm{P}$ \\
\hline Gibasis linearis (Benth.) Rohweder subsp. rhodantha (Torr.) D. R. Hunt & HAG-632 & $\mathrm{h}$ & $\mathrm{T}$ & A & $\mathrm{P}$ \\
\hline Gibasis pellucida (M. Martens \& Galeotti) D.R. Hunt & HAG-466 & $\mathrm{h}$ & $\mathrm{T}$ & A & $\mathrm{P}$ \\
\hline Tinantia erecta (Jacq.) Schltdl. & HAG-38 & $\mathrm{h}$ & $\mathrm{T}$ & A & A \\
\hline Tradescantia commelinioides Schult. \& Schult. f. & HAG-493 & $\mathrm{h}$ & $\mathrm{T}$ & A & $\mathrm{P}$ \\
\hline Tradescantia crassifolia Cav. subsp. crassifolia & HAG-593 & $\mathrm{h}$ & $\mathrm{T}$ & A & $\mathrm{P}$ \\
\hline \multicolumn{6}{|l|}{ CYPERACEAE } \\
\hline Carex leucocarpa Boeckeler & HAG-490 & $\mathrm{h}$ & $\mathrm{T}$ & A & $\mathrm{P}$ \\
\hline Carex longissima M.E. Jones & AAR-11117 & $\mathrm{h}$ & $\mathrm{T}$ & A & $\mathrm{P}$ \\
\hline Carex turbinata Liebm. & AAR-11119 & $\mathrm{h}$ & $\mathrm{T}$ & A & $\mathrm{P}$ \\
\hline Carex sp. & & $\mathrm{h}$ & $\mathrm{T}$ & A & $\mathrm{P}$ \\
\hline Cyperus hermaphroditus (Jacq.) Standl. & HAG-485 & $\mathrm{h}$ & $\mathrm{T}$ & A & $\mathrm{P}$ \\
\hline Cyperus manimae Kunth & HAG-486 & $\mathrm{h}$ & $\mathrm{T}$ & A & $\mathrm{P}$ \\
\hline Cyperus pallidicolor (Kük.) G.C. Tucker & HAG-36 & $\mathrm{h}$ & $\mathrm{T}$ & A & $\mathrm{P}$ \\
\hline Cyperus perusillus Boeckeler & HAG-522 & $\mathrm{h}$ & $\mathrm{T}$ & A & $\mathrm{P}$ \\
\hline Cyperus sp. 1 & HAG-487 & $\mathrm{h}$ & $\mathrm{T}$ & A & $\mathrm{P}$ \\
\hline Cyperus sp. 2 & HAG-556 & $\mathrm{h}$ & $\mathrm{T}$ & A & $\mathrm{P}$ \\
\hline \multicolumn{6}{|l|}{ DIOSCOREACEAE } \\
\hline Dioscorea jaliscana S. Watson & HAG-616 & $\mathrm{h}$ & $\mathrm{T}$ & A & $\mathrm{P}$ \\
\hline \multicolumn{6}{|l|}{ HYPOXIDACEAE } \\
\hline Hypoxis decumbens L. & HAG-542 & $\mathrm{h}$ & $\mathrm{T}$ & A & $\mathrm{P}$ \\
\hline Hypoxis mexicana Schult. \& Schult. f. & HAG-477 & $\mathrm{h}$ & $\mathrm{T}$ & A & $\mathrm{P}$ \\
\hline \multicolumn{6}{|l|}{ IRIDACEAE } \\
\hline Nemastylis tenuis (Herb.) S. Watson & HAG-619 & $\mathrm{h}$ & $\mathrm{T}$ & A & $\mathrm{P}$ \\
\hline Sisyrinchium pringlei B.L. Rob. \& Greenm. & HAG-14 & $\mathrm{h}$ & $\mathrm{T}$ & A & $\mathrm{P}$ \\
\hline Sisyrinchium sp. & HAG-516 & $\mathrm{h}$ & $\mathrm{R}$ & A & $\mathrm{P}$ \\
\hline Tigridia estelae López-Ferr. \& Espejo & HAG-597 & $\mathrm{h}$ & $\mathrm{T}$ & A & $\mathrm{P}$ \\
\hline \multicolumn{6}{|l|}{ JUNCACEAE } \\
\hline Juncus tenuis Willd. & HAG-489 & $\mathrm{h}$ & $\mathrm{T}$ & A & $\mathrm{P}$ \\
\hline
\end{tabular}




\begin{tabular}{|c|c|c|c|c|c|}
\hline & Cols. $/ \mathbf{N}^{\circ}$ de colecta & $\mathbf{A}$ & $\mathbf{H}$ & $\mathbf{N}$ & $\mathbf{C}$ \\
\hline \multicolumn{6}{|l|}{ LILIACEAE } \\
\hline Calochortus venustulus Greene var. venustulus & HAG-578 & $\mathrm{h}$ & $\mathrm{T}$ & A & $\mathrm{P}$ \\
\hline \multicolumn{6}{|l|}{ ORCHIDACEAE } \\
\hline Bletia ensifolia L.O. Williams $\dagger$ & HAG-601 & $\mathrm{h}$ & $\mathrm{T}$ & A & $\mathrm{P}$ \\
\hline Bletia macristhmochila Greenm. & HAG-5 & $\mathrm{h}$ & $\mathrm{T}, \mathrm{R}$ & A & $\mathrm{P}$ \\
\hline Bletia purpurata A. Rich. \& Galeotti & HAG-793 & $\mathrm{h}$ & $\mathrm{T}$ & A & $\mathrm{P}$ \\
\hline Bletia santosii sp. nov. $* \bullet \dagger$ & HAG-472 & $\mathrm{h}$ & $\mathrm{R}$ & A & $\mathrm{P}$ \\
\hline Bletia villae Soto Arenas $\uparrow$ & HAG-439 & $\mathrm{h}$ & $\mathrm{T}$ & A & $\mathrm{P}$ \\
\hline Corallorhiza maculata (Raf.) Raf. $\dagger$ & HAG-582 & $\mathrm{h}$ & $\mathrm{T}$ & $\mathrm{O}$ & $\mathrm{P}$ \\
\hline Deiregyne eriophora (Rob. \& Greenm.) Garay $\dagger$ & HAG-415 & $\mathrm{h}$ & $\mathrm{T}$ & A & $\mathrm{P}$ \\
\hline Dichromanthus aurantiacus (La Llave \& Lex.) Salazar \& Soto Arenas & HAG-473 & $\mathrm{h}$ & $\mathrm{T}, \mathrm{R}$ & A & $\mathrm{P}$ \\
\hline Dichromanthus michuacanus (Lex.) Salazar, Soto Arenas \& Hágsater $\dagger$ & HAG-211 & $\mathrm{h}$ & $\mathrm{T}$ & A & $\mathrm{P}$ \\
\hline Encyclia adenocarpa (Lex.) Schltr. & HAG-481 & $\mathrm{h}$ & $\mathrm{E}$ & A & $\mathrm{P}$ \\
\hline Encyclia aenicta Dressler \& G.E. Pollard & JGG-2230 & $\mathrm{h}$ & $\mathrm{E}$ & A & $\mathrm{P}$ \\
\hline Epidendrum falcatum Lindl. & PVR-88 & $\mathrm{h}$ & $\mathrm{R}$ & A & $\mathrm{P}$ \\
\hline Epidendrum sp. & Nat. & $\mathrm{h}$ & $\mathrm{R}$ & A & $\mathrm{P}$ \\
\hline Goodyera striata Rchb. f. & HAG-405 & $\mathrm{h}$ & $\mathrm{T}$ & A & $\mathrm{P}$ \\
\hline $\begin{array}{l}\text { Greenwoodiella micrantha (Lex.) Salazar \& R. Jiménez var. garayana (R. González) Salazar } \\
\& \text { R. Jiménez } \dagger\end{array}$ & HAG-345 & $\mathrm{h}$ & $\mathrm{T}$ & A & $\mathrm{P}$ \\
\hline Habenaria filifera S.Watson & HAG-23 & $\mathrm{h}$ & $\mathrm{T}$ & A & $\mathrm{P}$ \\
\hline Habenaria macvaughiana R. González $\dagger$ & HAG-575 & $\mathrm{h}$ & $\mathrm{T}$ & A & $\mathrm{P}$ \\
\hline Isochilus bracteatus (Lex) Salazar \& Soto Arenas ex Espejo \& López-Ferr. $\dagger$ & PVR-89 & $\mathrm{h}$ & $\mathrm{R}, \mathrm{E}$ & A & $\mathrm{P}$ \\
\hline Liparis madrensis Soto Arenas, Salazar \& R. Jiménez $\dagger$ & HAG-790 & $\mathrm{h}$ & $\mathrm{T}$ & A & $\mathrm{P}$ \\
\hline Malaxis brachyrrhynchos (Rchb. f.) Ames $\dagger$ & HAG-460 & $\mathrm{h}$ & $\mathrm{T}$ & A & $\mathrm{P}$ \\
\hline Malaxis maianthemifolia Schltdl. \& Cham. $\dagger$ & HAG-446 & $\mathrm{h}$ & $\mathrm{T}$ & A & $\mathrm{P}$ \\
\hline Malaxis pringlei (S. Watson) Ames $\dagger$ & HAG-626 & $\mathrm{h}$ & $\mathrm{T}$ & A & $\mathrm{P}$ \\
\hline Malaxis tepicana Ames $\dagger$ & HAG-527 & $\mathrm{h}$ & $\mathrm{T}$ & A & $\mathrm{P}$ \\
\hline Malaxis thlaspiformis A. Rich. \& Galeotti & CPD-8 & $\mathrm{h}$ & $\mathrm{T}$ & A & $\mathrm{P}$ \\
\hline Oncidium brachyandrum Lindl. & HAG-414 & $\mathrm{h}$ & $\mathrm{E}$ & A & $\mathrm{P}$ \\
\hline Prosthechea squalida (Lex.) Soto Arenas \& Salazar & HAG-410 & $\mathrm{h}$ & $\mathrm{E}$ & A & $\mathrm{P}$ \\
\hline Rhynchostele aptera (Lex.) Soto Arenas \& Salazar & HAG-408 & $\mathrm{h}$ & $\mathrm{E}$ & A & $\mathrm{P}$ \\
\hline Rhynchostele cervantesii (La Llave \& Lex.) Soto Arenas \& Salazar & HAG-387 & $\mathrm{h}$ & $\mathrm{E}$ & A & $\mathrm{P}$ \\
\hline Schiedeella albovaginata (C. Schweinf.) Burns-Bal. & HAG-774 & $\mathrm{h}$ & $\mathrm{T}$ & A & $\mathrm{P}$ \\
\hline Stelis xerophila (Schltr.) Soto Arenas & PVR-87 & $\mathrm{h}$ & $\mathrm{R}, \mathrm{E}$ & A & $\mathrm{P}$ \\
\hline \multicolumn{6}{|l|}{ POACEAE } \\
\hline Digitaria filiformis (L.) Koeler $\dagger$ & HAG-630 & $\mathrm{h}$ & $\mathrm{T}$ & A & $\mathrm{P}$ \\
\hline Lasiacis ruscifolia (Kunth) Hitchc. & HAG-292 & $\mathrm{h}$ & $\mathrm{T}$ & A & $\mathrm{P}$ \\
\hline Muhlenbergia alamosae Vasey & HAG-164 & $\mathrm{h}$ & $\mathrm{R}$ & A & $\mathrm{P}$ \\
\hline Muhlenbergia brevifolia Scribn. ex Beal & DJP-12893 & $\mathrm{h}$ & $\mathrm{T}$ & A & $\mathrm{P}$ \\
\hline Muhlenbergia cenchroides (Humb. \& Bonpl. ex Willd.) P.M. Peterson $\uparrow$ & HAG-335 & $\mathrm{h}$ & $\mathrm{T}$ & A & $\mathrm{P}$ \\
\hline Muhlenbergia emersleyi Vasey & HAG-355a & $\mathrm{h}$ & $\mathrm{T}$ & A & $\mathrm{P}$ \\
\hline Muhlenbergia gigantea (E. Fourn.) Hitchc. & HAG-301 & $\mathrm{h}$ & $\mathrm{T}$ & A & $\mathrm{P}$ \\
\hline Muhlenbergia michisensis Y. Herrera \& P.M. Peterson † & HAG-266 & $\mathrm{h}$ & $\mathrm{T}$ & A & $\mathrm{P}$ \\
\hline Muhlenbergia quadridentata (Kunth) Kunth $\dagger$ & HAG-355b & $\mathrm{h}$ & $\mathrm{T}$ & A & $\mathrm{P}$ \\
\hline Muhlenbergia tenuifolia (Kunth) Kunth $\uparrow$ & HAG-293 & $\mathrm{h}$ & $\mathrm{T}$ & A & $\mathrm{P}$ \\
\hline
\end{tabular}




\begin{tabular}{|c|c|c|c|c|c|}
\hline & Cols. $/ N^{\circ}$ de colecta & $\mathbf{A}$ & $\mathbf{H}$ & $\mathbf{N}$ & $\mathbf{C}$ \\
\hline Muhlenbergia uniseta (Lag.) Columbus & HAG-623 & $\mathrm{h}$ & $\mathrm{T}$ & A & A \\
\hline Oplismenus burmannii (Retz.) P. Beauv. & HAG-627 & $\mathrm{h}$ & $\mathrm{T}$ & A & A \\
\hline Panicum hirticaule J. Presl & HAG-531 & $\mathrm{h}$ & $\mathrm{T}$ & A & A \\
\hline Peyritschia deyeuxioides (Kunth) Finot $\uparrow$ & HAG-350 & $\mathrm{h}$ & $\mathrm{T}$ & A & $\mathrm{P}$ \\
\hline Poa aпnиa $\mathrm{L}$. & HAG-628 & $\mathrm{h}$ & $\mathrm{T}$ & A & A \\
\hline Tripsacum zopilotense Hern.-Xol. \& Randolph & HAG-515 & $\mathrm{h}$ & $\mathrm{T}$ & A & $\mathrm{P}$ \\
\hline Trisetum palmeri Hitchc. & HAG-262 & $\mathrm{h}$ & $\mathrm{T}$ & A & $\mathrm{P}$ \\
\hline Trisetum viride (Kunth) Kunth & HAG-352 & $\mathrm{h}$ & $\mathrm{T}$ & A & $\mathrm{P}$ \\
\hline Zuloagaea bulbosa (Kunth) Bess & HAG-533 & $\mathrm{h}$ & $\mathrm{T}$ & A & $\mathrm{P}$ \\
\hline \multicolumn{6}{|l|}{ SMILACACEAE } \\
\hline Smilax moranensis M. Martens \& Galeotti & HAG-379 & $\mathrm{h}$ & $\mathrm{T}$ & A & $\mathrm{P}$ \\
\hline \multicolumn{6}{|l|}{ EUDICOTILEDÓNEAS } \\
\hline \multicolumn{6}{|l|}{ ACANTHACEAE } \\
\hline Hypoestes phyllostachya Baker & Nat. & $\mathrm{h}$ & $\mathrm{T}$ & A & $\mathrm{P}$ \\
\hline Pseuderanthemum praecox (Benth.) Leonard & HAG-380 & $\mathrm{h}$ & $\mathrm{T}$ & A & $\mathrm{P}$ \\
\hline \multicolumn{6}{|l|}{ ADOXACEAE } \\
\hline Sambucus nigra L. subsp. canadensis (L.) Bolli & Nat. & $\mathrm{a}$ & $\mathrm{T}$ & A & $\mathrm{P}$ \\
\hline \multicolumn{6}{|l|}{ AMARANTHACEAE } \\
\hline Gomphrena serrata $\mathrm{L}$. & RVA-2930 & $\mathrm{h}$ & $\mathrm{T}$ & A & $\mathrm{A}, \mathrm{P}$ \\
\hline Iresine diffusa Humb. \& Bonpl. ex Willd. var. diffusa & HAG-300 & h & $\mathrm{T}$ & A & A \\
\hline \multicolumn{6}{|l|}{ ANACARDIACEAE } \\
\hline Rhus aromatica Aiton var. schmidelioides (Schlecht.) Engl. & HAG-276 & ar & $\mathrm{T}$ & A & $\mathrm{P}$ \\
\hline \multicolumn{6}{|l|}{ APIACEAE } \\
\hline Coulterophytum macrophyllum J.M. Coult. \& Rose & HAG-510 & $\mathrm{h}$ & $\mathrm{T}$ & A & $\mathrm{P}$ \\
\hline Donnellsmithia juncea (Humb. \& Bonpl. ex Spreng.) Mathias \& Constance & HAG-521 & $\mathrm{h}$ & $\mathrm{T}$ & A & $\mathrm{P}$ \\
\hline Donnellsmithia ternata (S. Watson) Mathias \& Constance $\uparrow$ & HAG-115 & $\mathrm{h}$ & $\mathrm{T}$ & A & $\mathrm{P}$ \\
\hline Eryngium mexiae Constance & HAG-128 & $\mathrm{h}$ & $\mathrm{T}$ & A & $\mathrm{P}$ \\
\hline Micropleura renifolia Lag. & HAG-553 & $\mathrm{h}$ & $\mathrm{T}$ & A & $\mathrm{P}$ \\
\hline Prionosciadium sp. & HAG-544 & $\mathrm{h}$ & $\mathrm{T}$ & A & $\mathrm{P}$ \\
\hline \multicolumn{6}{|l|}{ APOCYNACEAE } \\
\hline Asclepias angustifolia Schweigg. & HAG-192 & $\mathrm{h}$ & $\mathrm{T}$ & A & $\mathrm{P}$ \\
\hline Asclepias atroviolacea Woodson & HAG-19 & $\mathrm{h}$ & $\mathrm{T}$ & A & $\mathrm{P}$ \\
\hline Vinca major $\mathrm{L}$. & Nat. & $\mathrm{h}$ & $\mathrm{T}$ & A & $\mathrm{P}$ \\
\hline \multicolumn{6}{|l|}{ AQUIFOLIACEAE } \\
\hline Ilex dugesii Fernald & HAG-122 & a & $\mathrm{T}$ & A & $\mathrm{P}$ \\
\hline \multicolumn{6}{|l|}{ ARALIACEAE } \\
\hline Aralia humilis Cav. & HAG-722 & a & $\mathrm{T}$ & A & $\mathrm{P}$ \\
\hline Dendropanax arboreus (L.) Decne. \& Planch. & HAG-297 & a, ar & $\mathrm{T}$ & A & $\mathrm{P}$ \\
\hline Oreopanax echinops (Schltdl. \& Cham.) Decne. \& Planch. & HAG-186 & $\mathrm{a}, \mathrm{ar}$ & $\mathrm{T}$ & A & $\mathrm{P}$ \\
\hline Oreopanax peltatus Linden ex Regel & HAG-251 & a & $\mathrm{T}$ & A & $\mathrm{P}$ \\
\hline \multicolumn{6}{|l|}{ ASTERACEAE } \\
\hline Acourtia patens (A. Gray) Reveal \& R.M. King & HAG-287 & $\mathrm{h}$ & $\mathrm{T}$ & A & $\mathrm{P}$ \\
\hline Ageratina bellidifolia (Benth.) R.M. King \& H. Rob. & HAG-188 & $\mathrm{h}$ & $\mathrm{T}$ & A & $\mathrm{P}$ \\
\hline Ageratina mairetiana (DC.) R.M. King \& H. Rob. var. elucens (McVaugh) B. L. Turner & DJP-12881 & ar & $\mathrm{T}$ & A & $\mathrm{P}$ \\
\hline Ageratina pichinchensis (Kunth) R.M. King \& H. Rob. & ACS-22594 & h, ar & $\mathrm{T}$ & A & $\mathrm{P}$ \\
\hline
\end{tabular}




\begin{tabular}{|c|c|c|c|c|c|}
\hline & Cols. $/ \mathbf{N}^{\circ}$ de colecta & $\mathbf{A}$ & $\mathbf{H}$ & $\mathbf{N}$ & $\mathbf{C}$ \\
\hline Ageratum corymbosum Zucc. ex Pers. & HAG-89 & $\mathrm{h}$ & $\mathrm{T}$ & A & $\mathrm{P}$ \\
\hline Aldama ensifolia (Sch. Bip.) E.E. Schill. \& Panero & HAG-254 & ar & $\mathrm{T}$ & A & $\mathrm{P}$ \\
\hline Aldama latibracteata (Hemsl.) E.E. Schill. \& Panero & HAG-147 & $\mathrm{h}$ & $\mathrm{T}$ & A & $\mathrm{P}$ \\
\hline Aldama parkinsonii (Hemsl.) E.E. Schill. \& Panero & HAG-137 & $\mathrm{h}$ & $\mathrm{T}$ & A & $\mathrm{P}$ \\
\hline Aldama pringlei (B. L. Rob. \& Greenm.) E.E. Schill. \& Panero & HAG-332 & $\mathrm{h}$ & $\mathrm{T}$ & A & $\mathrm{P}$ \\
\hline Aldama seemannii (Sch. Bip.) E.E. Schill. \& Panero & ACS-1611 & $\mathrm{h}$ & $\mathrm{T}$ & A & $\mathrm{P}$ \\
\hline Alloispermum palmeri (S. Watson ex A. Gray) C. F. Fernández \& Urbatsch var. palmeri & HAG-535 & $\mathrm{h}$ & $\mathrm{T}$ & A & $\mathrm{P}$ \\
\hline Baccharis heterophylla Kunth & HAG-418 & ar & $\mathrm{T}$ & A & $\mathrm{P}$ \\
\hline Bidens lemmonii A. Gray & HAG-558 & $\mathrm{h}$ & $\mathrm{R}$ & A & A \\
\hline Bidens mollifolia Sherff & HAG-532 & $\mathrm{h}$ & $\mathrm{T}$ & A & $\mathrm{P}$ \\
\hline Bidens ostruthioides (DC.) Sch. Bip. & HAG-119 & $\mathrm{h}$ & $\mathrm{T}$ & A & $\mathrm{P}$ \\
\hline Bidens sp. 1 & HAG-26a & $\mathrm{h}$ & $\mathrm{T}$ & A & A \\
\hline Bidens sp. 2 & HAG-26b & $\mathrm{h}$ & $\mathrm{T}$ & A & A \\
\hline Brickellia secundiflora (Lag.) A. Gray & HAG-165 & ar & $\mathrm{T}$ & A & $\mathrm{P}$ \\
\hline Chionolaena salicifolia (Bertol.) G.L. Nesom & HAG-264 & $\mathrm{h}$ & $\mathrm{T}$ & A & $\mathrm{P}$ \\
\hline Chromolaena ovaliflora (Hook. \& Arn.) R. M. King \& H. Rob. & HAG-128 & $\mathrm{h}$ & $\mathrm{T}$ & A & $\mathrm{P}$ \\
\hline Cirsium anartiolepis Petr. & HAG-226 & $\mathrm{h}$ & $\mathrm{T}$ & A & $\mathrm{P}$ \\
\hline Coreocarpus congregatus (S.F. Blake) E.B. Sm. & HAG-604 & $\mathrm{h}$ & $\mathrm{R}$ & A & A \\
\hline Coreopsis petrophila A. Gray & HAG-53 & $\mathrm{h}$ & $\mathrm{R}$ & A & $\mathrm{P}$ \\
\hline Cosmos carvifolius Benth. & HAG-8 & $\mathrm{h}$ & $\mathrm{T}$ & A & $\mathrm{P}$ \\
\hline Cosmos crithmifolius Kunth & ACS-4401 & ar & $\mathrm{T}$ & A & $\mathrm{P}$ \\
\hline Cosmos linearifolius (Sch. Bip.) Hemsl. & HAG-238 & $\mathrm{h}$ & $\mathrm{T}$ & A & $\mathrm{P}$ \\
\hline Cosmos palmeri B. L. Rob. & HAG-492 & $\mathrm{h}$ & $\mathrm{T}$ & A & $\mathrm{P}$ \\
\hline Cosmos parviflorus (Jacq.) Pers. & HAG-612 & $\mathrm{h}$ & $\mathrm{T}$ & A & A \\
\hline Dahlia coccinea Cav. & HAG-523 & $\mathrm{h}$ & $\mathrm{T}$ & A & $\mathrm{P}$ \\
\hline Dahlia rupicola P. D. Sørensen & HAG-557 & ar & $\mathrm{R}$ & $\mathrm{A}$ & $\mathrm{P}$ \\
\hline Dahlia sherffii P. D. Sørensen & HAG-581 & $\mathrm{h}$ & $\mathrm{R}$ & A & $\mathrm{P}$ \\
\hline Erigeron polycephalus (Larsen) G.L. Nesom & HAG-283 & $\mathrm{h}$ & $\mathrm{T}$ & A & $\mathrm{P}$ \\
\hline Erigeron subacaulis (McVaugh) G.L. Nesom & LL-636 & $\mathrm{h}$ & $\mathrm{T}$ & A & $\mathrm{P}$ \\
\hline Galinsoga parviflora $\mathrm{Cav}$. & HAG-98 & $\mathrm{h}$ & $\mathrm{T}$ & A & A \\
\hline Guardiola mexicana Humb. \& Bonpl. var. mexicana & HAG-177 & $\mathrm{h}$ & $\mathrm{T}$ & A & $\mathrm{P}$ \\
\hline Hieracium fendleri Sch. Bip. subsp. fendleri & HAG-356 & $\mathrm{h}$ & $\mathrm{T}$ & A & $\mathrm{P}$ \\
\hline Hofmeisteria gayleana B.L. Turner & HAG-372 & $\mathrm{h}$ & $\mathrm{T}$ & A & $\mathrm{P}$ \\
\hline Hymenostephium cordatum (Hook. \& Arn.) S.F. Blake & DJP-12941 & $\mathrm{h}$ & $\mathrm{T}$ & A & $\mathrm{P}$ \\
\hline Iostephane heterophylla (Cav.) Hemsl. & HAG-39 & $\mathrm{h}$ & $\mathrm{T}$ & A & $\mathrm{P}$ \\
\hline Jaegeria hirta (Lag.) Less. & HAG-561 & $\mathrm{h}$ & $\mathrm{T}$ & A & A \\
\hline Lagascea helianthifolia Kunth & HAG-234 & $\mathrm{h}$ & $\mathrm{T}$ & A & $\mathrm{P}$ \\
\hline Leibnitzia lyrata (Sch.Bip.) G.L. Nesom & HAG-365 & $\mathrm{h}$ & $\mathrm{T}$ & A & $\mathrm{P}$ \\
\hline Melampodium perfoliatum (Cav.) Kunth & HAG-145 & $\mathrm{h}$ & $\mathrm{T}$ & A & A \\
\hline Montanoa leucantha (Lag.) S.F. Blake subsp. arborescens V. A. Funk & HAG-142 & ar & $\mathrm{T}$ & A & $\mathrm{P}$ \\
\hline Perityle turneri A.M. Powell & ACS-4919 & $\mathrm{h}$ & $\mathrm{T}$ & A & $\mathrm{P}$ \\
\hline Pinaropappus roseus (Less.) Less. var. roseus & HAG-343 & $\mathrm{h}$ & $\mathrm{T}$ & A & $\mathrm{P}$ \\
\hline Piptothrix areolaris (DC.) R.M. King \& H. Rob & HAG-49 & $\mathrm{h}$ & $\mathrm{T}$ & A & $\mathrm{P}$ \\
\hline Piqueria triflora Hemsl. & HAG-166 & $\mathrm{h}$ & $\mathrm{T}$ & A & A \\
\hline Podachaenium eminens (Lag.) Sch. Bip. & HAG-339 & ar & $\mathrm{T}$ & A & $\mathrm{P}$ \\
\hline
\end{tabular}




\begin{tabular}{|c|c|c|c|c|c|}
\hline & Cols./No de colecta & $\mathbf{A}$ & $\mathbf{H}$ & $\mathbf{N}$ & $\mathbf{C}$ \\
\hline Porophyllum coloratum (Kunth) DC. var. coloratum & HAG-193 & $\mathrm{h}$ & $\mathrm{T}$ & A & $\mathrm{A}, \mathrm{P}$ \\
\hline Psacalium pachyphyllum (Sch. Bip.) Rydb. & HAG-134 & $\mathrm{h}$ & $\mathrm{T}$ & A & $\mathrm{P}$ \\
\hline Psacalium poculiferum (S. Watson) Rydb. & HAG-45 & $\mathrm{h}$ & $\mathrm{T}$ & A & $\mathrm{P}$ \\
\hline Pseudognaphalium attenuatum (DC.) Anderb. var. attenuatum & HAG-347 & $\mathrm{h}$ & $\mathrm{T}$ & A & $\mathrm{P}$ \\
\hline Pseudognaphalium inornatum (DC.) Anderb. & RVA-2963 & $\mathrm{h}$ & $\mathrm{T}$ & A & $\mathrm{P}$ \\
\hline Roldana barba-johannis (DC.) H. Rob. \& Brettell & HAG-270 & ar & $\mathrm{T}$ & A & $\mathrm{P}$ \\
\hline Roldana gonzaleziae (B.L. Turner) B.L. Turner $\dagger$ & HAG-80 & $\mathrm{h}$ & $\mathrm{T}$ & A & $\mathrm{P}$ \\
\hline Roldana hartwegii (Benth.) H. Rob. \& Brettell & HAG-104 & $\mathrm{h}$, ar & $\mathrm{T}$ & A & $\mathrm{P}$ \\
\hline Roldana jurgensenii (Hemsl.) H. Rob. \& Brettell $\uparrow$ & HAG-342 & $\mathrm{h}$, ar & $\mathrm{T}$ & A & $\mathrm{P}$ \\
\hline Roldana subpeltata (Sch. Bip.) H. Rob. \& Brettell & HAG-178 & $\mathrm{h}$ & $\mathrm{T}$ & A & $\mathrm{P}$ \\
\hline Sinclairia palmeri (A. Gray) B.L. Turner & HAG-602 & $\mathrm{h}$ & $\mathrm{T}$ & A & $\mathrm{P}$ \\
\hline Stevia elatior Kunth & HAG-138 & $\mathrm{h}$ & $\mathrm{T}$ & A & $\mathrm{P}$ \\
\hline Stevia jaliscensis B.L. Rob. & HAG-48 & $\mathrm{h}$ & $\mathrm{T}$ & A & $\mathrm{P}$ \\
\hline Stevia lucida Lag. & HAG-196 & $\mathrm{h}$ & $\mathrm{T}$ & A & $\mathrm{P}$ \\
\hline Stevia micradenia B.L. Rob. & RDW-7931 & $\mathrm{h}$ & $\mathrm{T}$ & A & $\mathrm{P}$ \\
\hline Stevia micrantha Lag. & HAG-483 & $\mathrm{h}$ & $\mathrm{T}$ & A & A \\
\hline Stevia nelsonii B.L. Rob. & RDW-7936 & $\mathrm{h}$ & $\mathrm{T}$ & A & $\mathrm{P}$ \\
\hline Tagetes filifolia Lag. & GDG-5 & $\mathrm{h}$ & $\mathrm{T}$ & A & A \\
\hline Taraxacum officinale F.H. Wigg. & Nat. & $\mathrm{h}$ & $\mathrm{T}$ & A & $\mathrm{P}$ \\
\hline Tithonia calva Sch. Bip. var. calva & HAG-373 & ar & $\mathrm{T}$ & A & $\mathrm{P}$ \\
\hline Trigonospermum annuum McVaugh \& Lask. & ACS-22571 & $\mathrm{h}$, ar & $\mathrm{T}$ & A & A \\
\hline Trigonospermum melampodioides DC. & HAG-245 & $\mathrm{h}$ & $\mathrm{T}$ & A & $\mathrm{P}$ \\
\hline Verbesina cymbipalea Blake $\uparrow$ & HAG-148 & $\mathrm{h}$ & $\mathrm{T}$ & A & $\mathrm{P}$ \\
\hline Verbesina grayii (Sch. Bip.) Benth. ex Hemsl. & HAG-361 & $\mathrm{h}$ & $\mathrm{T}$ & A & $\mathrm{P}$ \\
\hline Verbesina sp. & HAG-518 & h & $\mathrm{T}$ & A & $\mathrm{P}$ \\
\hline Wedelia tegetis Strother & HAG-52 & $\mathrm{h}$ & $\mathrm{T}$ & A & $\mathrm{P}$ \\
\hline \multicolumn{6}{|l|}{ BEGONIACEAE } \\
\hline Begonia biserrata Lindl. & HAG-511 & h & $\mathrm{T}$ & A & $\mathrm{P}$ \\
\hline Begonia gracilis Kunth & HAG-35 & $\mathrm{h}$ & $\mathrm{T}, \mathrm{R}$ & A & $\mathrm{P}$ \\
\hline Begonia tapatia Burt-Utley \& McVaugh & HAG-536 & $\mathrm{h}$ & $\mathrm{T}, \mathrm{R}$ & A & $\mathrm{P}$ \\
\hline \multicolumn{6}{|l|}{ BETULACEAE } \\
\hline Alnus jorullensis Kunth subsp. jorullensis & HAG-62 & a & $\mathrm{T}$ & A & $\mathrm{P}$ \\
\hline Alnus jorullensis Kunth subsp. lutea Furlow & HAG-254 & $\mathrm{a}$ & $\mathrm{T}$ & A & $\mathrm{P}$ \\
\hline Ostrya virginiana (Mill.) K. Koch var. virginiana & HAG.294 & a & $\mathrm{T}$ & A & $\mathrm{P}$ \\
\hline \multicolumn{6}{|l|}{ BRASSICACEAE } \\
\hline Lepidium virginicum $\mathrm{L}$. & Nat. & $\mathrm{h}$ & $\mathrm{T}$ & A & A \\
\hline \multicolumn{6}{|l|}{ CACTACEAE } \\
\hline Disocactus speciosus (Cav.) Barthlott subsp. speciosus (Cav.) Barthlott & HAG-212 & $\mathrm{h}$ & $\mathrm{R}$ & A & $\mathrm{P}$ \\
\hline Echinocereus acifer (Otto ex Salm-Dick) Haage & HAG-156 & $\mathrm{g}$ & $\mathrm{R}$ & A & $\mathrm{P}$ \\
\hline Mammillaria senilis G. Lodd. ex Salm-Dick & HAG-205 & $\mathrm{g}$ & $\mathrm{R}$ & A & $\mathrm{P}$ \\
\hline Opuntia preciadoae Scheinvar, Olalde, Gallegos \& J. Morales & HAG-631 & ar & $\mathrm{T}, \mathrm{R}$ & A & $\mathrm{P}$ \\
\hline Opuntia sp. 1 & HAG-160 & ar & $\mathrm{T}, \mathrm{R}$ & A & $\mathrm{P}$ \\
\hline Opuntia sp. 2 & HAG-210 & ar & $\mathrm{T}, \mathrm{R}$ & A & $\mathrm{P}$ \\
\hline \multicolumn{6}{|l|}{ CALCEOLARIACEAE } \\
\hline Calceolaria mexicana Benth. subsp. mexicana & HAG-388 & $\mathrm{h}$ & $\mathrm{T}$ & A & A \\
\hline
\end{tabular}




\begin{tabular}{|c|c|c|c|c|c|}
\hline & Cols. $/ N^{\circ}$ de colecta & $\mathbf{A}$ & $\mathbf{H}$ & $\mathbf{N}$ & $\mathbf{C}$ \\
\hline \multicolumn{6}{|l|}{ CAMPANULACEAE } \\
\hline Lobelia goldmanii (Fernald) T.J. Ayers & HAG-236 & $\mathrm{h}$ & $\mathrm{T}, \mathrm{R}$ & A & A \\
\hline Lobelia laxiflora Kunth var. laxiflora & HAG-221 & ar & $\mathrm{T}$ & A & $\mathrm{P}$ \\
\hline Lobelia macrocentron (Benth.) T.J. Ayers $\bullet \dagger$ & HAG-252 & $\mathrm{h}$ & $\mathrm{T}, \mathrm{R}$ & A & $\mathrm{P}$ \\
\hline Lobelia sinaloae Sprague & HAG-11 & $\mathrm{h}$ & $\mathrm{T}$ & A & A \\
\hline \multicolumn{6}{|l|}{ CAPRIFOLIACEAE } \\
\hline Lonicera pilosa (Kunth) Spreng. & HAG-173 & ar & $\mathrm{T}$ & A & $\mathrm{P}$ \\
\hline Valeriana deltoidea F. G. Mey. $\dagger$ & HAG-451 & $\mathrm{h}$ & $\mathrm{T}$ & A & $\mathrm{P}$ \\
\hline Valeriana sorbifolia Kunth & HAG-32 & $\mathrm{h}$ & $\mathrm{T}$ & A & $\mathrm{P}$ \\
\hline Valeriana urticifolia Kunth & HAG-17 & $\mathrm{h}$ & $\mathrm{T}$ & A & $\mathrm{P}$ \\
\hline \multicolumn{6}{|l|}{ CARYOPHYLLACEAE } \\
\hline Arenaria lanuginosa (Michx.) Rohrb. var. lanuginosa & HAG-363 & $\mathrm{h}$ & $\mathrm{T}$ & A & $\mathrm{P}$ \\
\hline Cerastium texanum Britton & HAG-170 & $\mathrm{h}$ & $\mathrm{T}$ & A & A, $\mathrm{P}$ \\
\hline Drymaria leptophylla (Cham. \& Schltdl.) Fenzl † & HAG-621 & $\mathrm{h}$ & $\mathrm{T}$ & A & A \\
\hline Drymaria villosa Cham. \& Schltdl. subsp. villosa & HAG-559 & $\mathrm{h}$ & $\mathrm{T}$ & A & A, $\mathrm{P}$ \\
\hline Stellaria cuspidata Willd. ex Schltdl. † & HAG-399 & $\mathrm{h}$ & $\mathrm{T}$ & A & $\mathrm{P}$ \\
\hline \multicolumn{6}{|l|}{ CISTACEAE } \\
\hline Helianthemum concolor (L. Riley) G. Ortega & DJP-12877 & $\mathrm{h}$ & $\mathrm{T}$ & A & $\mathrm{P}$ \\
\hline Helianthemum glomeratum Lag. ex DC. & HAG-187 & $\mathrm{h}$ & $\mathrm{T}$ & A & $\mathrm{P}$ \\
\hline \multicolumn{6}{|l|}{ CLETHRACEAE } \\
\hline Clethra hartwegii Britton & HAG-201 & a & $\mathrm{T}$ & A & $\mathrm{P}$ \\
\hline \multicolumn{6}{|l|}{ CONVOLVULACEAE } \\
\hline Ipomoea decemcornuta $\mathrm{O}^{\prime}$ Donell $\uparrow$ & HAG-611 & $\mathrm{h}$ & $\mathrm{T}$ & A & A \\
\hline Ipomoea orizabensis (Pelletan) Ledeb. ex Steud var. orizabensis & HAG-227 & $\mathrm{h}$ & $\mathrm{T}$ & A & $\mathrm{P}$ \\
\hline Ipomoea plummerae A. Gray & HAG-41 & $\mathrm{h}$ & $\mathrm{T}$ & A & $\mathrm{P}$ \\
\hline Ipomoea purpurea (L.) Roth & Nat. & $\mathrm{h}$ & $\mathrm{T}$ & A & A \\
\hline Ipomoea ternifolia $\mathrm{Cav}$. var. ternifolia & HAG-599 & $\mathrm{h}$ & $\mathrm{T}$ & A & A \\
\hline Ipomoea sp. & HAG-524 & $\mathrm{h}$ & $\mathrm{T}$ & A & A \\
\hline \multicolumn{6}{|l|}{ CORNACEAE } \\
\hline Cornus disciflora Moc. \& Sessé ex DC. & HAG-334 & $\mathrm{a}$ & $\mathrm{T}$ & A & $\mathrm{P}$ \\
\hline \multicolumn{6}{|l|}{ CRASSULACEAE } \\
\hline Echeveria affinis E. Walther & HAG-321 & $\mathrm{r}$ & $\mathrm{R}$ & A & $\mathrm{P}$ \\
\hline Echeveria dactylifera $\mathrm{E}$. Walther & HAG-131 & $\mathrm{r}$ & $\mathrm{T}, \mathrm{R}, \mathrm{E}$ & A & $\mathrm{P}$ \\
\hline Sedum jaliscanum S. Watson & HAG-620 & $\mathrm{h}$ & $\mathrm{R}$ & A & $\mathrm{P}$ \\
\hline Sedum quadripetalum R.T. Clausen & HAG-540 & $\mathrm{h}$ & $\mathrm{R}$ & A & $\mathrm{P}$ \\
\hline \multicolumn{6}{|l|}{ CUCURBITACEAE } \\
\hline Cyclanthera langaei Cogn. & HAG-499 & $\mathrm{h}$ & $\mathrm{T}$ & A & A \\
\hline Cyclanthera minima (S. Watson) Kearns \& C.E. Jones & HAG-614 & $\mathrm{h}$ & $\mathrm{T}$ & A & A \\
\hline Cyclanthera ribiflora (Schltdl.) Cogn. & HAG-617 & $\mathrm{h}$ & $\mathrm{T}$ & A & A \\
\hline \multicolumn{6}{|l|}{ ERICACEAE } \\
\hline Arbutus bicolor S. González, M. González \& P.D. Sørensen & HAG-255 & $\mathrm{a}$ & $\mathrm{T}$ & A & $\mathrm{P}$ \\
\hline Arbutus tessellata P. D. Sørensen & HAG-73 & $\mathrm{a}$ & $\mathrm{T}$ & A & $\mathrm{P}$ \\
\hline Arbutus xalapensis Kunth & HAG-209 & $\mathrm{a}$ & $\mathrm{T}$ & A & $\mathrm{P}$ \\
\hline Chimaphila maculata (L.) Pursh & KR-2 & $\mathrm{h}$ & $\mathrm{T}$ & A & $\mathrm{P}$ \\
\hline Comarostaphylis cf. polifolia (Kunth) Zucc. ex Klotzsch & HAG-784 & ar & $\mathrm{T}$ & A & $\mathrm{P}$ \\
\hline
\end{tabular}




\begin{tabular}{|c|c|c|c|c|c|}
\hline & Cols./No de colecta & $\mathbf{A}$ & $\mathbf{H}$ & $\mathbf{N}$ & $\mathbf{C}$ \\
\hline Gaultheria erecta Vent. & HAG-359 & ar & $\mathrm{T}$ & A & $\mathrm{P}$ \\
\hline Gaultheria myrsinoides Kunth & HAG-420 & ar & $\mathrm{T}$ & A & $\mathrm{P}$ \\
\hline \multicolumn{6}{|l|}{ EUPHORBIACEAE } \\
\hline Acalypha subviscida $\mathrm{S}$. Watson & HAG-517 & ar & $\mathrm{T}$ & A & $\mathrm{P}$ \\
\hline Croton flavescens Greenm. & HAG-508 & $\mathrm{a}$ & $\mathrm{T}$ & A & $\mathrm{P}$ \\
\hline Croton morifolius Willd. & HAG-461 & ar & $\mathrm{T}$ & A & $\mathrm{P}$ \\
\hline Euphorbia brachycera Engelm. & HAG-199 & $\mathrm{h}$ & $\mathrm{T}$ & A & $\mathrm{P}$ \\
\hline \multicolumn{6}{|l|}{ FABACEAE } \\
\hline Acaciella angustissima (Mill.) Britton \& Rose var. angustissima & HAG-719 & a & $\mathrm{T}$ & A & $\mathrm{P}$ \\
\hline Aeschynomene unijuga (M.E. Jones) Rudd & HAG-99 & $\mathrm{h}$ & $\mathrm{T}$ & A & $\mathrm{P}$ \\
\hline Astragalus sinaloae Barneby & HAG-570 & $\mathrm{h}$ & $\mathrm{T}$ & A & $\mathrm{P}$ \\
\hline Astragalus vaccarum A. Gray & ACS-21205 & $\mathrm{h}$ & $\mathrm{T}$ & A & $\mathrm{P}$ \\
\hline Calliandra grandiflora (L'Hér.) Benth. & HAG-600 & $\mathrm{a}$, ar & $\mathrm{T}$ & A & $\mathrm{P}$ \\
\hline Canavalia villosa Benth. & HAG-298 & $\mathrm{h}$ & $\mathrm{T}$ & A & $\mathrm{P}$ \\
\hline Cologania broussonetii (Balb.) DC. & HAG-567 & $\mathrm{h}$ & $\mathrm{T}$ & A & $\mathrm{P}$ \\
\hline Cologania cordata Fearing ex McVaugh & HAG-605a & $\mathrm{h}$ & $\mathrm{T}$ & A & $\mathrm{P}$ \\
\hline Cologania obovata Schltdl. & HAG-443 & $\mathrm{h}$ & $\mathrm{T}$ & A & $\mathrm{P}$ \\
\hline Crotalaria longirostrata Hook. \& Arn. & HAG-424 & $\mathrm{h}$ & $\mathrm{T}$ & A & $\mathrm{P}$ \\
\hline Crotalaria micans Link & HAG-141 & $\mathrm{h}$ & $\mathrm{T}$ & A & $\mathrm{P}$ \\
\hline Crotalaria polyphylla L. Riley & HAG-69 & $\mathrm{h}$ & $\mathrm{T}$ & A & $\mathrm{P}$ \\
\hline Dalea versicolor Zucc. & HAG-200 & $\mathrm{h}$ & $\mathrm{T}$ & A & $\mathrm{P}$ \\
\hline Desmodium bellum (S. F. Blake) B. G. Schub. & HAG-232 & $\mathrm{h}$, ar & $\mathrm{T}$ & A & $\mathrm{P}$ \\
\hline Desmodium cordistipulum Hemsl. & HAG-96 & $\mathrm{h}$ & $\mathrm{T}$ & A & $\mathrm{P}$ \\
\hline Desmodium densiflorum Hemsl. & HAG-285 & $\mathrm{h}$ & $\mathrm{T}$ & A & $\mathrm{P}$ \\
\hline Desmodium grahamii A. Gray & HAG-110 & $\mathrm{h}$ & $\mathrm{T}$ & A & $\mathrm{P}$ \\
\hline Desmodium molliculum (Kunth) DC. & HAG-588 & $\mathrm{h}$ & $\mathrm{T}$ & A & $\mathrm{P}$ \\
\hline Desmodium tortuosum (Sw.) DC. & HAG-569 & $\mathrm{h}$ & $\mathrm{T}$ & A & $\mathrm{P}$ \\
\hline Eriosema pulchellum (Kunth) G. Don & ACS-4907 & h, ar & $\mathrm{T}$ & A & $\mathrm{P}$ \\
\hline Hosackia alamosana Rose & HAG-92 & $\mathrm{h}$ & $\mathrm{T}$ & A & $\mathrm{P}$ \\
\hline Hosackia repens G. Don & HAG-337 & $\mathrm{h}$ & $\mathrm{T}$ & A & $\mathrm{P}$ \\
\hline Hosackia sp. & HAG-290 & $\mathrm{h}$ & $\mathrm{T}$ & A & $\mathrm{P}$ \\
\hline Lupinus chihuahuensis S. Watson $\dagger$ & HAG-329 & $\mathrm{h}$ & $\mathrm{T}$ & A & $\mathrm{P}$ \\
\hline Lupinus exaltatus Zucc. $\uparrow$ & HAG-249 & $\mathrm{h}$ & $\mathrm{T}$ & A & $\mathrm{P}$ \\
\hline Marina nutans (Cav.) Barneby & HAG-46 & ar & $\mathrm{T}$ & A & $\mathrm{P}$ \\
\hline Phaseolus coccineus L. & HAG-37 & $\mathrm{h}$ & $\mathrm{T}$ & A & $\mathrm{P}$ \\
\hline Phaseolus jaliscanus Piper & HAG-587 & $\mathrm{h}$ & $\mathrm{T}$ & A & $\mathrm{P}$ \\
\hline Phaseolus parvulus Greene & HAG-111a & $\mathrm{h}$ & $\mathrm{T}$ & A & A, P \\
\hline Phaseolus pauciflorus Sessé \& Moc. ex G. Don & HAG-590 & $\mathrm{h}$ & $\mathrm{T}$ & A & $\mathrm{P}$ \\
\hline Phaseolus vulgaris $\mathrm{L}$. & HAG-171 & $\mathrm{h}$ & $\mathrm{T}$ & A & A \\
\hline Senna foetidissima var. grandiflora (Benth.) H.S. Irwin \& Barneby & HAG-785 & ar & $\mathrm{T}$ & A & $P$ \\
\hline Trifolium amabile Kunth & HAG-525 & $\mathrm{h}$ & $\mathrm{T}$ & A & $\mathrm{P}$ \\
\hline Vachellia cornigera (L.) Seigler \& Ebinger & HAG-720 & ar & $\mathrm{T}$ & A & $\mathrm{P}$ \\
\hline Vachellia farnesiana (L.) Wight \& Arn. & HAG-725 & $\mathrm{a}, \mathrm{ar}$ & $\mathrm{T}$ & A & $\mathrm{P}$ \\
\hline Vachellia pennatula (Schltdl. \& Cham.) Seigler \& Ebinger & HAG-718 & $\mathrm{a}$ & $\mathrm{T}$ & A & $\mathrm{P}$ \\
\hline
\end{tabular}




\begin{tabular}{|c|c|c|c|c|c|}
\hline & Cols. $/ \mathbf{N}^{\circ}$ de colecta & $\mathbf{A}$ & $\mathbf{H}$ & $\mathbf{N}$ & $\mathbf{C}$ \\
\hline \multicolumn{6}{|l|}{ FAGACEAE } \\
\hline Quercus brachystachys Benth. & HAG-419 & a & $\mathrm{T}$ & A & $\mathrm{P}$ \\
\hline Quercus calophylla Schltdl. \& Cham. & HAG-278 & $\mathrm{a}$ & $\mathrm{T}$ & A & $\mathrm{P}$ \\
\hline Quercus castanea Née & HAG-552 & a & $\mathrm{T}$ & A & $\mathrm{P}$ \\
\hline Quercus convallata Trel. & HAG-724 & $\mathrm{a}$ & $\mathrm{T}$ & A & $\mathrm{P}$ \\
\hline Quercus fulva Liebm. & HAG-754 & a & $\mathrm{T}$ & A & $\mathrm{P}$ \\
\hline Quercus gentryi C. H. Müll. & HAG-247 & $\mathrm{a}$ & $\mathrm{T}$ & A & $\mathrm{P}$ \\
\hline Quercus grahamii Benth. $\dagger$ & HAG-431 & $\mathrm{a}$ & $\mathrm{T}$ & A & $\mathrm{P}$ \\
\hline Quercus laeta Liebm. & HAG-64 & a & $\mathrm{T}$ & A & $\mathrm{P}$ \\
\hline Quercus reticulata Bonpl. & HAG-120 & $\mathrm{a}$ & $\mathrm{T}$ & A & $\mathrm{P}$ \\
\hline Quercus rugosa Née & DJP-12934 & a & $\mathrm{T}$ & A & $\mathrm{P}$ \\
\hline Quercus scytophylla Liebm. & HAG-748 & $\mathrm{a}$ & $\mathrm{T}$ & A & $\mathrm{P}$ \\
\hline Quercus splendens Née & HAG-717 & $\mathrm{a}$ & $\mathrm{T}$ & A & $\mathrm{P}$ \\
\hline Quercus viminea Trel. & HAG-435 & $\mathrm{a}$ & $\mathrm{T}$ & A & $\mathrm{P}$ \\
\hline Quercus sp. 1 & HAG-761 & a & $\mathrm{T}$ & A & $\mathrm{P}$ \\
\hline Quercus sp. 2 & HAG-608 & $\mathrm{a}$ & $\mathrm{T}$ & A & $\mathrm{P}$ \\
\hline \multicolumn{6}{|l|}{ GARRYACEAE } \\
\hline Garrya laurifolia Hartw. ex Benth. subsp. laurifolia & HAG-744 & a & $\mathrm{T}$ & A & $\mathrm{P}$ \\
\hline Garrya longifolia Rose & HAG-275 & $\mathrm{a}$ & $\mathrm{T}$ & A & $\mathrm{P}$ \\
\hline \multicolumn{6}{|l|}{ GENTIANACEAE } \\
\hline Gentiana mirandae Paray $\dagger$ & HAG-259 & $\mathrm{h}$ & $\mathrm{R}$ & A & $\mathrm{P}$ \\
\hline Gentianella amarella $(\mathrm{L}$.$) Boerner$ & HAG-109 & $\mathrm{h}$ & $\mathrm{T}$ & A & $\mathrm{A}, \mathrm{P}$ \\
\hline Gentianopsis lanceolata (Benth.) Iltis & HAG-112 & $\mathrm{h}$ & $\mathrm{T}$ & A & A \\
\hline Halenia schiedeana Griseb. & HAG-534 & $\mathrm{h}$ & $\mathrm{T}$ & A & A \\
\hline Zeltnera wigginsii (Broome) G. Mans. & HAG-635 & $\mathrm{h}$ & $\mathrm{T}$ & A & A \\
\hline \multicolumn{6}{|l|}{ GERANIACEAE } \\
\hline Geranium lilacinum $\mathrm{R}$. Knuth $\uparrow$ & HAG-316 & $\mathrm{h}$ & $\mathrm{T}$ & A & $\mathrm{P}$ \\
\hline Geranium seemannii Peyr. & HAG-161 & $\mathrm{h}$ & $\mathrm{T}$ & A & $\mathrm{P}$ \\
\hline Geranium wislizeni $\mathrm{S}$. Watson & DJP-12892 & $\mathrm{h}$ & $\mathrm{T}$ & A & $\mathrm{P}$ \\
\hline \multicolumn{6}{|l|}{ GESNERIACEAE } \\
\hline Achimenes antirrhina (DC.) C.V. Morton & HAG-502 & $\mathrm{h}$ & $\mathrm{R}$ & A & $\mathrm{P}$ \\
\hline Moussonia jaliscana (S.Watson) D. L. Denham ex Ram.-Roa $\dagger$ & HAG-197 & $\mathrm{h}$ & $\mathrm{R}$ & A & $\mathrm{P}$ \\
\hline \multicolumn{6}{|l|}{ HYDRANGEACEAE } \\
\hline Philadelphus karwinskyanus Koehne & HAG-336 & ar & $\mathrm{T}$ & A & $\mathrm{P}$ \\
\hline \multicolumn{6}{|l|}{ HYPERICACEAE } \\
\hline Hypericum pauciflorum Kunth & HAG-546 & $\mathrm{h}$ & $\mathrm{T}$ & A & $\mathrm{P}$ \\
\hline Hypericum silenoides Juss. & HAG-530 & $\mathrm{h}$ & $\mathrm{T}$ & A & $\mathrm{A}, \mathrm{P}$ \\
\hline \multicolumn{6}{|l|}{ LAMIACEAE } \\
\hline Cunila pycnantha B.L. Rob. \& Greenm. & ACC-4486 & ar, h & $\mathrm{T}$ & A & $\mathrm{P}$ \\
\hline Hyptis urticoides Kunth & HAG-82 & $\mathrm{h}$ & $\mathrm{T}$ & A & $\mathrm{P}$ \\
\hline Lepechinia caulescens (Ortega) Epling & HAG-591 & $\mathrm{h}$ & $\mathrm{T}$ & A & $\mathrm{P}$ \\
\hline Salvia crucis Epling & HAG-354 & $\mathrm{h}$ & $\mathrm{T}$ & A & $\mathrm{P}$ \\
\hline Salvia elegans Vahl & HAG-144 & $\mathrm{h}$ & $\mathrm{T}$ & A & $\mathrm{P}$ \\
\hline Salvia gesneriiflora Lindl. \& Paxton & HAG-734 & ar & $\mathrm{T}$ & A & $\mathrm{P}$ \\
\hline Salvia hispanica L. & HAG-146 & $\mathrm{h}$ & $\mathrm{T}$ & A & A \\
\hline Salvia iodantha Fernald & HAG-102 & $\mathrm{h}$ & $\mathrm{T}$ & A & $\mathrm{P}$ \\
\hline
\end{tabular}




\begin{tabular}{|c|c|c|c|c|c|}
\hline & Cols. $/ \mathbf{N}^{\circ}$ de colecta & $\mathbf{A}$ & $\mathbf{H}$ & $\mathbf{N}$ & $\mathbf{C}$ \\
\hline Salvia lavanduloides Kunth & HAG-100 & $\mathrm{h}$ & $\mathrm{T}$ & A & $\mathrm{P}$ \\
\hline Salvia mexicana $\mathrm{L}$. & HAG-124 & $\mathrm{h}$, ar & $\mathrm{T}$ & A & $\mathrm{P}$ \\
\hline Salvia microphylla Kunth & Nat. & ar, h & $\mathrm{T}$ & A & $\mathrm{P}$ \\
\hline Salvia prunelloides Kunth & HAG-106 & $\mathrm{h}$ & $\mathrm{T}$ & A & $\mathrm{P}$ \\
\hline Salvia roscida Fernald & HAG-243 & $\mathrm{h}$ & $\mathrm{T}$ & A & $\mathrm{P}$ \\
\hline Salvia sphacelifolia Epling & HAG-721 & ar & $\mathrm{T}$ & A & $\mathrm{P}$ \\
\hline Scutellaria dumetorum Schltdl. $\uparrow$ & HAG-498 & $\mathrm{h}$ & $\mathrm{T}$ & A & $\mathrm{P}$ \\
\hline Stachys coccinea Ortega & HAG-237 & $\mathrm{h}$, ar & $\mathrm{T}$ & A & $\mathrm{P}$ \\
\hline \multicolumn{6}{|l|}{ LENTIBULARIACEAE } \\
\hline Pinguicula oblongiloba A. DC. & HAG-442 & $\mathrm{h}$ & $\mathrm{R}$ & A & $\mathrm{P}$ \\
\hline Pinguicula parvifolia B. L. Rob. $\dagger$ & HAG-440 & $\mathrm{h}$ & $\mathrm{R}$ & A & $\mathrm{P}$ \\
\hline \multicolumn{6}{|l|}{ LORANTHACEAE } \\
\hline Cladocolea cupulata Kuijt & HAG-179 & ar & $\mathrm{E}$ & M & $\mathrm{P}$ \\
\hline Cladocolea mcvaughii Kuijt & HAG-225 & ar & $\mathrm{E}$ & M & $\mathrm{P}$ \\
\hline Psittacanthus calyculatus (DC.) G. Don & HAG-169 & ar & $\mathrm{E}$ & M & $\mathrm{P}$ \\
\hline \multicolumn{6}{|l|}{ LYTHRACEAE } \\
\hline Cuphea appendiculata Benth. & HAG-284 & ar & $\mathrm{T}$ & A & $\mathrm{P}$ \\
\hline Cuphea hookeriana Walp. & HAG-88 & $\mathrm{h}$ & $\mathrm{T}$ & A & $\mathrm{P}$ \\
\hline Cuphea ownbeyi S.A. Graham & HAG-788 & $\mathrm{h}$ & $\mathrm{T}$ & A & $\mathrm{P}$ \\
\hline Cuphea tolucana Peyr. $\dagger$ & HAG-787 & $\mathrm{h}$ & $\mathrm{T}$ & A & $\mathrm{P}$ \\
\hline Cuphea watsoniana Koehne & DJP-12867 & $\mathrm{h}$ & $\mathrm{T}$ & A & $\mathrm{P}$ \\
\hline \multicolumn{6}{|l|}{ MALPIGHIACEAE } \\
\hline Galphimia floribunda C.E. Anderson & HAG-248 & ar & $\mathrm{T}$ & A & $\mathrm{P}$ \\
\hline \multicolumn{6}{|l|}{ MALVACEAE } \\
\hline Pseudabutilon orientale (Standl. \& Steyerm.) Fryxell & HAG-239 & $\mathrm{h}$ & $\mathrm{T}$ & A & $\mathrm{P}$ \\
\hline Sida rhombifolia L. & HAG-44 & $\mathrm{h}$ & $\mathrm{T}$ & A & $\mathrm{P}$ \\
\hline Tilia americana $\mathrm{L}$. & HAG-464 & $\mathrm{a}$ & $\mathrm{T}$ & A & $\mathrm{P}$ \\
\hline Triumfetta chihuahuensis Standl. & HAG-610 & ar & $\mathrm{T}$ & A & $\mathrm{P}$ \\
\hline \multicolumn{6}{|l|}{ MELASTOMATACEAE } \\
\hline Monochaetum calcaratum (DC.) Triana & ACS-4359 & ar & $\mathrm{T}$ & A & $P$ \\
\hline Tibouchina almedae Todzia & HAG-93 & $\mathrm{h}$ & $\mathrm{T}$ & A & $\mathrm{p}$ \\
\hline \multicolumn{6}{|l|}{ MELIACEAE } \\
\hline Cedrela odorata $\mathrm{L}$. & HAG-386 & $\mathrm{a}$ & $\mathrm{T}$ & A & $\mathrm{P}$ \\
\hline \multicolumn{6}{|l|}{ MYRTACEAE } \\
\hline Eugenia crenularis Lundell & HAG-383 & ar & $\mathrm{T}$ & A & $\mathrm{P}$ \\
\hline \multicolumn{6}{|l|}{ NYCTAGINACEAE } \\
\hline Mirabilis jalapa $\mathrm{L}$. & Nat. & $\mathrm{h}$ & $\mathrm{T}$ & A & $\mathrm{P}$ \\
\hline \multicolumn{6}{|l|}{ OLEACEAE } \\
\hline Fraxinus sp. & HAG-739 & $\mathrm{a}$ & $\mathrm{T}$ & A & $\mathrm{P}$ \\
\hline \multicolumn{6}{|l|}{ ONAGRACEAE } \\
\hline Fuchsia arborescens Sims & HAG-244 & ar & $\mathrm{T}$ & A & $\mathrm{P}$ \\
\hline Fuchsia cylindracea Lindl. & HAG-13 & ar & $\mathrm{T}$ & A & $\mathrm{P}$ \\
\hline Fuchsia thymifolia Kunth subsp. thymifolia & HAG-586 & ar & $\mathrm{T}$ & A & $\mathrm{P}$ \\
\hline Lopezia miniata Lag. ex DC. & HAG-132 & $\mathrm{h}$ & $\mathrm{T}$ & A & $\mathrm{P}$ \\
\hline Lopezia ovata (Plitman, Raven \& Breedlove) Plitmann, P.H. Raven \& Breedlove & HAG-315 & $\mathrm{h}$ & $\mathrm{T}$ & A & $\mathrm{P}$ \\
\hline
\end{tabular}




\begin{tabular}{|c|c|c|c|c|c|}
\hline & Cols. $/ \mathbf{N}^{\circ}$ de colecta & $\mathbf{A}$ & $\mathbf{H}$ & $\mathbf{N}$ & $\mathbf{C}$ \\
\hline Lopezia semeiandra Plitmann, P.H. Raven \& Breedlove & DJP-12912 & $\mathrm{h}$ & $\mathrm{T}$ & A & $\mathrm{P}$ \\
\hline Oenothera rosea L'Hér. ex Aiton & HAG-367 & $\mathrm{h}$ & $\mathrm{T}$ & A & $\mathrm{A}, \mathrm{P}$ \\
\hline \multicolumn{6}{|l|}{ OROBANCHACEAE } \\
\hline Buchnera obliqua Benth. & RDW-7928 & $\mathrm{h}$ & $\mathrm{T}$ & M & $\mathrm{P}$ \\
\hline Castilleja arvensis Schltdl. \& Cham. & HAG-280 & $\mathrm{h}$ & $\mathrm{T}$ & M & A \\
\hline Castilleja chlorosceptron G.L. Nesom & HAG-549 & $\mathrm{h}$ & $\mathrm{T}$ & M & $\mathrm{P}$ \\
\hline Castilleja holmgrenii J.M. Egger & L-24316a-c & $\mathrm{h}$ & $\mathrm{T}$ & M & $\mathrm{P}$ \\
\hline Castilleja nervata Eastw. & HAG-331 & $\mathrm{h}$ & $\mathrm{T}$ & M & $\mathrm{P}$ \\
\hline Castilleja ortegae Standl. & HAG-260 & $\mathrm{h}$ & $\mathrm{T}$ & M & $\mathrm{P}$ \\
\hline Castilleja spiranthoides Standl. & HAG-406 & $\mathrm{h}$ & $\mathrm{T}$ & M & $\mathrm{P}$ \\
\hline Castilleja tenuiflora Benth. & HAG-279 & $\mathrm{h}$ & $\mathrm{T}$ & M & $\mathrm{P}$ \\
\hline Conopholis alpina Liebm. & HAG-437 & $\mathrm{h}$ & $\mathrm{T}$ & $\mathrm{O}$ & $\mathrm{P}$ \\
\hline Lamourouxia longiflora Benth. & HAG-265 & $\mathrm{h}$ & $\mathrm{T}$ & M & $\mathrm{P}$ \\
\hline Lamourouxia multifida Kunth & HAG-231 & $\mathrm{h}$ & $\mathrm{T}$ & M & $\mathrm{P}$ \\
\hline Lamourouxia viscosa Kunth & HAG-230 & $\mathrm{h}$ & $\mathrm{T}$ & M & $\mathrm{P}$ \\
\hline Pedicularis glabra McVaugh \& Mellich. $\uparrow$ & HAG-85 & $\mathrm{h}$ & $\mathrm{T}$ & M & $\mathrm{P}$ \\
\hline Seymeria virgata (Kunth) Benth. & HAG-480 & $\mathrm{h}$ & $\mathrm{T}$ & M & $\mathrm{P}$ \\
\hline \multicolumn{6}{|l|}{ OXALIDACEAE } \\
\hline Oxalis alpina (Rose) Rose ex R. Knuth & HAG-584 & $\mathrm{h}$ & $\mathrm{T}$ & A & $\mathrm{P}$ \\
\hline Oxalis corniculata $\mathrm{L}$. & Nat. & $\mathrm{h}$ & $\mathrm{T}$ & A & $\mathrm{P}$ \\
\hline Oxalis divergens Benth. $\dagger$ & HAG-478 & $\mathrm{h}$ & $\mathrm{T}$ & A & $\mathrm{P}$ \\
\hline Oxalis hernandesii DC. & HAG-583 & $\mathrm{h}$ & $\mathrm{T}$ & A & $\mathrm{P}$ \\
\hline Oxalis latifolia Kunth & HAG-462 & $\mathrm{h}$ & $\mathrm{R}$ & A & $\mathrm{P}$ \\
\hline \multicolumn{6}{|l|}{ PAPAVERACEAE } \\
\hline Argemone ochroleuca Sweet & Nat. & $\mathrm{h}$ & $\mathrm{T}$ & A & $\mathrm{A}, \mathrm{P}$ \\
\hline Bocconia arborea $\mathrm{S}$. Watson & HAG-731 & a & $\mathrm{T}$ & A & $\mathrm{P}$ \\
\hline \multicolumn{6}{|l|}{ PASSIFLORACEAE } \\
\hline Passiflora jorullensis Kunth $\dagger$ & HAG-789 & $\mathrm{h}$ & $\mathrm{T}$ & A & $\mathrm{P}$ \\
\hline \multicolumn{6}{|l|}{ PENTAPHYLACACEAE } \\
\hline Ternstroemia lineata DC. & HAG-758 & a, ar & $\mathrm{T}$ & A & $\mathrm{P}$ \\
\hline \multicolumn{6}{|l|}{ PHYTOLACCACEAE } \\
\hline Phytolacca icosandra $\mathrm{L}$. & HAG-153 & $\mathrm{h}$ & $\mathrm{T}$ & A & $\mathrm{P}$ \\
\hline \multicolumn{6}{|l|}{ PLANTAGINACEAE } \\
\hline Cymbalaria muralis G. Gaertn., B. Mey. \& Scherb. & Nat. & $\mathrm{h}$ & $\mathrm{R}$ & A & $\mathrm{P}$ \\
\hline Penstemon plagapineus Straw & HAG-83 & $\mathrm{h}$ & $\mathrm{T}$ & A & $\mathrm{P}$ \\
\hline Penstemon roseus (Cerv. ex Sweet) G. Don & HAG-69 & $\mathrm{h}$ & $\mathrm{T}$ & A & $\mathrm{P}$ \\
\hline Plantago australis Lam. subsp. hirtella (Kunth) Rahn & HAG-338 & $\mathrm{h}$ & $\mathrm{T}$ & A & $\mathrm{P}$ \\
\hline Russelia retrorsa Greene & HAG-176 & $\mathrm{h}$ & $\mathrm{T}$ & A & $\mathrm{P}$ \\
\hline Sibthorpia repens (Mutis ex L.) Kuntze & HAG-403 & $\mathrm{h}$ & $\mathrm{R}$ & A & $\mathrm{P}$ \\
\hline \multicolumn{6}{|l|}{ POLEMONIACEAE } \\
\hline Bonplandia geminiflora Cav. & HAG-125 & $\mathrm{h}$ & $\mathrm{T}$ & A & A \\
\hline Loeselia glandulosa (Cav.) Don subsp. glandulosa & HAG-250 & $\mathrm{h}$ & $\mathrm{T}$ & A & A \\
\hline Loeselia mexicana (Lam.) Brandegee & HAG-306 & ar & $\mathrm{T}$ & A & $\mathrm{P}$ \\
\hline \multicolumn{6}{|l|}{ POLYGALACEAE } \\
\hline Hebecarpa obscura (Benth.) J.R. Abbott & HAG-417 & $\mathrm{h}$ & $\mathrm{T}$ & A & $\mathrm{P}$ \\
\hline
\end{tabular}




\begin{tabular}{|c|c|c|c|c|c|}
\hline & Cols. $/ N^{\circ}$ de colecta & $\mathbf{A}$ & $\mathbf{H}$ & $\mathbf{N}$ & $\mathbf{C}$ \\
\hline Monnina ciliolata Sessé \& Moc. ex DC. & HAG-190 & $\mathrm{h}$, ar & $\mathrm{T}$ & A & $\mathrm{P}$ \\
\hline Polygala parryi A. W. Benn. $\dagger$ & HAG-416 & $\mathrm{h}$ & $\mathrm{T}$ & A & $\mathrm{P}$ \\
\hline Polygala subalata $\mathrm{S}$. Watson $\dagger$ & HAG-482 & $\mathrm{h}$ & $\mathrm{T}$ & A & A \\
\hline \multicolumn{6}{|l|}{ POLYGONACEAE } \\
\hline Rumex obtusifolius L. & Nat. & $\mathrm{h}$ & $\mathrm{T}$ & A & $P$ \\
\hline \multicolumn{6}{|l|}{ PRIMULACEAE } \\
\hline Anagallis arvensis $\mathrm{L}$. & HAG-154 & $\mathrm{h}$ & $\mathrm{T}$ & A & A \\
\hline \multicolumn{6}{|l|}{ RANUNCULACEAE } \\
\hline Aquilegia skinneri Hook. & JRC-12 & $\mathrm{h}$ & $\mathrm{T}$ & A & $\mathrm{P}$ \\
\hline Thalictrum gibbosum Lecoy. $\dagger$ & HAG-494 & $\mathrm{h}$ & $\mathrm{T}$ & A & $\mathrm{P}$ \\
\hline \multicolumn{6}{|l|}{ RHAMNACEAE } \\
\hline Ceanothus coeruleus Lag. & HAG-233 & $\mathrm{h}$, ar & $\mathrm{T}$ & A & $\mathrm{P}$ \\
\hline \multicolumn{6}{|l|}{ ROSACEAE } \\
\hline Cercocarpus macrophyllus C.K. Schneid. & HAG-273 & $\mathrm{a}$ & $\mathrm{T}$ & A & $\mathrm{P}$ \\
\hline Crataegus mexicana Moc. \& Sessé & HAG-409 & $\mathrm{a}$ & $\mathrm{T}$ & A & $\mathrm{P}$ \\
\hline Crataegus rosei Eggl. & Nat. & a & $\mathrm{T}$ & A & $\mathrm{P}$ \\
\hline Lachemilla sibbaldiifolia (Kunth) Rydb. & HAG-327 & $\mathrm{h}$ & $\mathrm{T}$ & A & $\mathrm{P}$ \\
\hline Prunus serotina Ehrh. subsp. serotina & HAG-730 & $\mathrm{a}$ & $\mathrm{T}$ & A & $\mathrm{P}$ \\
\hline Rubus cymosus Rydb. $\dagger$ & HAG-528 & ar & $\mathrm{T}$ & A & $\mathrm{P}$ \\
\hline Rubus palmeri Rydb. & RR-13 & ar & $\mathrm{T}$ & A & $\mathrm{P}$ \\
\hline \multicolumn{6}{|l|}{ RUBIACEAE } \\
\hline Bouvardia tenuifolia Standl. & HAG-21 & $\mathrm{h}$ & $\mathrm{T}$ & A & $\mathrm{P}$ \\
\hline Bouvardia ternifolia (Cav.) Schltdl. & HAG-299 & $\mathrm{h}$, ar & $\mathrm{T}$ & A & $\mathrm{P}$ \\
\hline Chiococca sp. & ACC-4532 & ar & $\mathrm{T}$ & A & $\mathrm{P}$ \\
\hline Crusea coccinea DC. var. breviloba Loes. & HAG-496 & $\mathrm{h}$ & $\mathrm{T}$ & A & $\mathrm{P}$ \\
\hline Crusea longiflora (Willd. ex Roem. \& Schult.) W.R. Anderson & HAG-562 & $\mathrm{h}$ & $\mathrm{T}$ & A & A \\
\hline Crusea setosa (M. Martens \& Galeotti) Standl. \& Steyerm. & HAG-514 & $\mathrm{h}$ & $\mathrm{T}$ & A & A \\
\hline Deppea guerrerensis Dwyer \& Lorence & HAG-376 & ar & $\mathrm{T}$ & A & $\mathrm{P}$ \\
\hline Galium mexicanum Kunth & HAG-127 & $\mathrm{h}$ & $\mathrm{T}$ & A & $\mathrm{P}$ \\
\hline Hoffmannia cuneatissima B.L. Rob. & HAG-500 & ar & $\mathrm{T}$ & A & $\mathrm{P}$ \\
\hline Relbunium laevigatum (DC.) Hemsl. & HAG-195b & $\mathrm{h}$ & $\mathrm{T}$ & A & $\mathrm{P}$ \\
\hline Spermacoce suaveolens (G. Mey.) Kuntze & HAG-126 & $\mathrm{h}$ & $\mathrm{T}$ & A & $\mathrm{P}$ \\
\hline Spermacoce sp. & HAG-208 & $\mathrm{h}$ & $\mathrm{T}$ & A & $\mathrm{P}$ \\
\hline \multicolumn{6}{|l|}{ RUTACEAE } \\
\hline Peltostigma eximium C.V. Morton & HAG-377 & ar & $\mathrm{T}$ & A & $\mathrm{P}$ \\
\hline \multicolumn{6}{|l|}{ SABIACEAE } \\
\hline Meliosma dentata (Liebm.) Urb. & HAG-385 & $\mathrm{a}$ & $\mathrm{T}$ & A & $\mathrm{P}$ \\
\hline \multicolumn{6}{|l|}{ SANTALACEAE } \\
\hline Phoradendron bolleanum (Seem.) Eichler & HAG-219 & $\mathrm{h}$ & $\mathrm{E}$ & M & $\mathrm{P}$ \\
\hline Phoradendron longifolium Eichler ex Trel. & HAG-180 & ar & $\mathrm{E}$ & M & $\mathrm{P}$ \\
\hline \multicolumn{6}{|l|}{ SAXIFRAGACEAE } \\
\hline Heuchera wellsiae R. A. Folk & ACS-4938 & $\mathrm{h}$ & $\mathrm{T}$ & A & $\mathrm{P}$ \\
\hline \multicolumn{6}{|l|}{ SCROPHULARIACEAE } \\
\hline Buddleja cordata Kunth & HAG-736 & $\mathrm{a}$, ar & $\mathrm{T}$ & A & $\mathrm{P}$ \\
\hline Buddleja parviflora Kunth & HAG-150 & ar & $\mathrm{T}$ & A & $\mathrm{P}$ \\
\hline
\end{tabular}




\begin{tabular}{|c|c|c|c|c|c|}
\hline & Cols./No de colecta & $\mathbf{A}$ & $\mathbf{H}$ & $\mathbf{N}$ & $\mathrm{C}$ \\
\hline Buddleja sessiliflora Kunth & HAG-330 & ar & $\mathrm{T}$ & A & $\mathrm{P}$ \\
\hline \multicolumn{6}{|l|}{ SOLANACEAE } \\
\hline Cestrum thyrsoideum Kunth & HAG-123 & ar & $\mathrm{T}$ & A & $\mathrm{P}$ \\
\hline Jaltomata procumbens (Cav.) J.L. Gentry & HAG-504 & $\mathrm{h}$ & $\mathrm{T}$ & A & A \\
\hline Physalis philadelphica Lam. & HAG-107 & $\mathrm{h}$ & $\mathrm{T}$ & A & A \\
\hline Solanum americanum Mill. & Nat. & $\mathrm{h}$ & $\mathrm{T}$ & A & $\mathrm{P}$ \\
\hline Solanum appendiculatum Dunal & HAG-786 & $\mathrm{h}$ & $\mathrm{T}$ & A & $\mathrm{P}$ \\
\hline Solanum bulbocastanum Dunal & HAG-520 & $\mathrm{h}$ & $\mathrm{T}$ & A & A, P \\
\hline Solanum erianthum D. Don & HAG-598 & $\mathrm{h}$ & $\mathrm{T}$ & A & $\mathrm{P}$ \\
\hline Solanum ferrugineum Jacq. & HAG-729 & ar & $\mathrm{T}$ & A & $\mathrm{P}$ \\
\hline Solanum nigricans $\mathrm{M}$. Martens \& Galeotti & HAG-738 & ar & $\mathrm{T}$ & A & $\mathrm{P}$ \\
\hline Solanum stoloniferum Schltdl. & HAG-509 & $\mathrm{h}$ & $\mathrm{T}$ & A & $\mathrm{P}$ \\
\hline \multicolumn{6}{|l|}{ STYRACACEAE } \\
\hline Styrax ramirezii Greenm. & HAG-375 & $\mathrm{a}$ & $\mathrm{T}$ & A & $\mathrm{P}$ \\
\hline \multicolumn{6}{|l|}{ URTICACEAE } \\
\hline Pilea skutchii Killip & HAG-495 & $\mathrm{h}$ & $\mathrm{R}$ & A & $\mathrm{P}$ \\
\hline Urera corallina (Liebm.) Wedd. & HAG-465a & $\mathrm{a}$ & $\mathrm{T}$ & A & $\mathrm{P}$ \\
\hline \multicolumn{6}{|l|}{ VERBENACEAE } \\
\hline Citharexylum affine D. Don & HAG-274 & $\mathrm{a}$ & $\mathrm{T}$ & A & $\mathrm{P}$ \\
\hline Lippia umbellata Cav. & HAG-735 & ar & $\mathrm{T}$ & A & $\mathrm{P}$ \\
\hline Verbena carolina $\mathrm{L}$. & HAG-526 & $\mathrm{h}$ & $\mathrm{T}$ & A & $\mathrm{P}$ \\
\hline \multicolumn{6}{|l|}{ VIOLACEAE } \\
\hline Viola grahamii Benth. & HAG-322 & $\mathrm{h}$ & $\mathrm{T}$ & A & $\mathrm{P}$ \\
\hline Viola umbraticola Kunth & HAG-360 & $\mathrm{h}$ & $\mathrm{T}$ & A & $\mathrm{P}$ \\
\hline \multicolumn{6}{|l|}{ VITACEAE } \\
\hline Vitis cinerea (Engelm.) Millardet & ZLD-17 & $\mathrm{b}$ & $\mathrm{T}$ & A & $\mathrm{P}$ \\
\hline Vitis tiliifolia Humb. \& Bonpl. ex Schult. & HAG-430 & $\mathrm{b}$ & $\mathrm{T}$ & A & $\mathrm{P}$ \\
\hline
\end{tabular}

